\title{
Principal Curves for Lumen Center Extraction and Flow Channel Width Estimation in 3-D Arterial Networks: Theory, Algorithm, and Validation
}

\author{
Wilbur C. K. Wong, Ronald W. K. So, and Albert C. S. Chung
}

\begin{abstract}
We present an energy-minimization-based framework for locating the centerline and estimating the width of tubelike objects from their structural network with a nonparametric model. The nonparametric representation promotes simple modeling of nested branches and $\boldsymbol{n}$-way furcations, i.e., structures that abound in an arterial network, e.g., a cerebrovascular circulation. Our method is capable of extracting the entire vascular tree from an angiogram in a single execution with a proper initialization. A succinct initial model from the user with arterial network inlets, outlets, and branching points is sufficient for complex vasculature. The novel method is based upon the theory of principal curves. In this paper, theoretical extension to grayscale angiography is discussed, and an algorithm to find an arterial network as principal curves is also described. Quantitative validation on a number of simulated data sets, synthetic volumes of 19 BrainWeb vascular models, and 32 Rotterdam Coronary Artery volumes was conducted. We compared the algorithm to a state-of-the-art method and further tested it on two clinical data sets. Our algorithmic outputs-lumen centers and flow channel widths-are important to various medical and clinical applications, e.g., vasculature segmentation, registration and visualization, virtual angioscopy, and vascular atlas formation and population study.
\end{abstract}

Index Terms-Angiography, arterial networks, blood vessels, centerlines, principal curves.

\section{INTRODUCTION}

$\mathbf{T}$ HE diagnosis and the prognosis of a vascular disease very often rely on 3-D angiography. An angiographic image provides comprehensive information on a circulation of interest. The lumen image also allows a physician to better understand the disease pathology. An appropriate treatment can be then planned. To assess the operation's effectiveness, a posttreatment image is oftentimes acquired. Angiographic segmentation offers a physician access to patient-specific 3-D vascular models for effective and efficient clinical comprehension. The vessel centerline is as important as the segmentation. It is a piece of crucial information in advanced image analysis, processing and visualization, viz., virtual angioscopy [1], [2], population study

Manuscript received August 17, 2010; revised September 19, 2011; accepted November 09, 2011. Date of publication December 09, 2011; date of current version March 21, 2012. This work was supported by the K. S. Lo Foundation. The associate editor coordinating the review of this manuscript and approving it for publication was Prof. Sina Farsiu.

The authors are with the Lo Kwee-Seong Medical Image Analysis Laboratory, Department of Computer Science and Engineering, The Hong Kong University of Science and Technology (HKUST), Clear Water Bay, Hong Kong (e-mail: achung@cse.ust.hk).

Color versions of one or more of the figures in this paper are available online at http://ieeexplore.ieee.org.

Digital Object Identifier 10.1109/TIP.2011.2179054 of vessel attributes [3], real-time 3-D/2-D vascular registration [4], and vasculature visualization [5], [6]. Algorithms to extract vessel centerlines can be categorized into two classes, i.e., automatic and semiautomatic. The former requires no user intervention in producing the vessel centerlines. The latter needs at least a single user-supplied point to kick off the execution. An automatic approach usually relies on the postprocessing of the vascular segmentation [1], [2], [7], [8]. Nevertheless, if one wants to get a satisfactory centerline extraction, a topologically and morphologically correct segmentation (with no handles and cavities) is compulsory, which is indeed very difficult to obtain from clinical data. Semiautomatic algorithms based on a vessel tracer are capable of producing a centerline with subvoxel accuracy starting from a user-supplied point. These algorithms include a mathematical morphology-based method [9] and iterative tracking methods [10]-[12]. The vessel axis is traced from the user-defined point progressively. The direction of the next axial point is locally determined with structural analysis. Worz and Rohr [12] proposed to use a 3-D cylindrical intensity model with the Kalman filter for incremental segmentwise tracking of a single vessel. Florin et al. [10] exploited Monte Carlo sampler to enable tracking of branches at a furcation. In one of our previous works [11], we posed the tracking problem on a probabilistic framework to allow interactive user intervention during algorithmic execution.

Vessel tracking with a single point, however, is inadequate in applications where the user wants to control the destination point of the traced path. To solve this problem, methods that permit the user to supply both start point and endpoint were developed [13], [14]. The voxel lattice is treated as a graph, and the centerline tracing problem is reduced to the well-known shortest path problem. Wink et al. [13] and Li and Yezzi [14] added one extra nonspatial dimension (scale or lumen width) to the graph to make the tracking of the vessel's interior more accurate. The centerline extracted is a sequence of connected voxels; thus, the accuracy is only up to voxel level. Parametric or spline modeling of a vessel centerline can achieve subvoxel accuracy. However, the number of spline control points and the point locations have to be selected with care. Dense control points are necessary for extracting complex vasculature centerline, while a few points are good enough for mildly bended or straight vessel segment. Special implementations may be needed, depending on the spline type, if the user wants the spline to pass through certain locations. Moreover, extracting the centerlines from a vascular network with these algorithms is not trivial. Multiple executions are required. The number of executions is decided by the number of distal network outlets; a single execution gives 
a path from the arterial root to an outlet. In this paper, we propose a better design that entertains finding the entire vascular tree with a single execution, as well as subvoxel accuracy.

Our main contribution is to propose an energy-minimizationbased framework for locating the centerline and estimating the width of tubelike objects from their structural network with the nonparametric model. In the framework, we exploit nonparametric representation to model the vascular network. Nested branches and $n$-way furcations are expressed in a typical graph adjacency list. The user is required to define an initial model by clicking a few positions in the image volume. The amount of user interaction depends on the complexity of the vasculature. An algorithm based on the theory of principal curves is introduced to refine the initial model, find centerlines of the network, and determine the lumen (i.e., flow channel) widths in a single execution. An earlier version of this paper was presented in [15]. The closest work is the semiautomatic extraction algorithm in [16]. They exploited the B-spline curve to model the centerline of a single vessel segment. The number of spline control points has to be predefined in their method. The insertion of anchor points (locations where the solution must go through) is not trivial and is coupled with the complexity of the B-spline. This is because the spline control points are not on the curve itself, forcing the curve to pass through an anchor needs further computation.

\section{INTRODUCTION TO PRINCIPAL CURVES}

\section{A. Theory of Principal Curves}

Hastie and Stuetzle [17] defined the notion of principal curves in 1989. A curve that smoothly passes through the "middle" of the input points gives the best nonlinear input summary. Later, Kégl et al. [18] refined the concept of principal curves by incorporating a length constraint, making the principal curves exist for any input point distribution with finite second moments. Then, the diverse and practical usage of principal curves has received considerable attention. In image processing, selected pixels are commonly transformed into a plain point cloud (points without any associated attribute) with respect to a reference origin and the pixel size, permitting a direct usage of principal curves. Suppose $\mathbb{X} \subset \mathbb{R}^{d}$ is a set of vectors representing the coordinates of the selected pixels, where $d=2$ denotes the domain dimensionality of a 2-D image. A smooth curve $\mathbf{f}(t)=\left[f_{1}(t), \ldots, f_{d}(t)\right]^{T}$ parameterized by a real argument $t$ is a principal curve of $\mathbb{X}$ if:

1) $\mathbf{f}$ does not intersect itself, i.e., $a \neq b \Rightarrow \mathbf{f}(a) \neq \mathbf{f}(b)$;

2) $\mathbf{f}$ has a given length $L$ inside any bounded region in $\mathbb{R}^{d}$;

3) $\mathbf{f}$ is self-consistent, i.e., $\mathbf{f}(t)=E\left(\mathbf{X} \mid \mathbf{f}^{-1}(\mathbf{X})=t\right)$

where $\mathbf{X}$ is a random variable on $\mathbb{X}, \mathbf{f}^{-1}(\vec{x})$ is known as the projection index of a point $\vec{x}$ on curve $\mathbf{f}$, and

$$
\mathbf{f}^{-1}(\vec{x})=\sup _{t}\left\{t:\|\vec{x}-\mathbf{f}(t)\|=\inf _{\tau}\|\vec{x}-\mathbf{f}(\tau)\|\right\} .
$$

Operator $\|\cdot\|$ denotes $l^{2}$-norm in $\mathbb{R}^{d}$. This projection index takes a particular value of $t$, whose corresponding location on the principal curve $\mathbf{f}$ has the shortest orthogonal distance (denoted by inf $\|\cdot\|$ ) to point $\vec{x}$. If there exists more than one of such value, the maximum one is chosen to be the projection index (denoted by $\sup \{\cdot\}$ ). The self-consistency property of principal curves guarantees that every point on the principal curve coincides with the "middle" or expected location of all the input points that are closest to itself. To further elaborate this property, we determine the principal curve location $\mathbf{f}(t)$ by evaluating the expected value of the random variable $\mathbf{X}$ that satisfies condition $\mathbf{f}^{-1}(\mathbf{X})=t$. Suppose we know how to find the projection index $\mathbf{f}^{-1}(\vec{x})$; the expectation is computed as follows:

$$
\begin{aligned}
\mathbf{f}(t) & =E\left(\mathbf{X} \mid \mathbf{f}^{-1}(\mathbf{X})=t\right) \\
& =\sum_{\vec{x} \in \mathbb{X}_{t}} \vec{x} g_{\mathbf{X}}(\vec{x})
\end{aligned}
$$

where $\mathbb{X}_{t}=\left\{\vec{x}: \vec{x} \in \mathbb{X}, \mathbf{f}^{-1}(\vec{x})=t\right\}$ is a set of input points that are closest to $\mathbf{f}(t)$ and $g_{\mathbf{X}}(\vec{x})$ is the probability mass function (pmf) of the discrete random variable $\mathbf{X}$. Since we have no extra knowledge of the input points (they are plain), we obtain the following pmf:

$$
g_{\mathbf{X}}(\vec{x})=\left\{\begin{array}{lll}
1 /\left|\mathbb{X}_{t}\right|, & \text { for } & \vec{x} \in \mathbb{X}_{t} ; \\
0, & \text { for } & \vec{x} \in \mathbb{R}^{d} / \mathbb{X}_{t}
\end{array}\right.
$$

where operator $|\cdot|$ returns the cardinality of the argument set. Combining (2) and (3) leads to a conclusion that a point on a principal curve is the average of its projected points. This asserts the principal curves self-consistency property.

\section{B. Extension to Grayscale Angiograms}

Given an angiographic segmentation, the direct usage of principal curves can give satisfactory extraction of lumen centers; nonetheless, a correct segmentation is difficult to obtain. This makes the straightforward application impractical in a clinical environment. This section shows how to use principal curves to extract lumen centers from a grayscale angiogram without the need for segmentation. Without segmenting the vessels, all the image pixels have to be processed. To make the smooth curve sensitive to the lumens, the pmf in (2) should be proportional to a lumen existence probability instead of being uniformly distributed among all the points in $\mathbb{X}_{t}$. A viable option to estimate the existence probability is to exploit a lumen cross-sectional intensity model. One can approximate this probability with the correlation between the model and the observations. We therefore focus on the correlation-based approach. The object to be detected in this case is the lumen cross-sectional intensity profile. We define the intensity model as a function of $\|\vec{u}\|$, where $\vec{u}$ is the relative position vector of a point to the lumen center. The position giving the maximum correlation between this model and the observations is therefore at the lumen center. The pmf is given as follows:

$$
g_{\mathbf{X}}(\vec{x})=\frac{1}{Z} \int_{-a}^{a} I\left(\vec{v} x+v \hat{n}_{t}\right) h_{t}\left(\left\|v \hat{n}_{t}\right\|\right) d v
$$

where constant $Z$ is for normalization, constant $a$ defines the model span, $I(\cdot)$ gives the intensity of the argument position, and $h_{t}(\cdot)$ and $\hat{n}_{t}$ are the intensity model and the normal vector at $\mathbf{f}(t)$, respectively. This equation expresses the pmf of a 2-D problem; the model span is a finite line segment between $\mathbf{f}(t) \pm$ $a \hat{n}_{t}$. In case of the 3-D problem, the span is a normal disc at $\mathbf{f}(t)$ of radius $a$. In other words, we consider a pencil of normal vectors rather than one particular normal vector at $\mathbf{f}(t)$ in a 
3-D space. However, the computation of such a pmf is expensive. It involves evaluation of a definite integral, which leads to numerous image interpolations. Furthermore, pmf precomputation at every pixel location is not possible because the integrand depends on the normal of the smooth curve. We show in Appendix A that this pmf $g_{\mathbf{X}}(\vec{x})$ is expected to give a maximum value at the lumen center under Rician and Gaussian noise.

We also find that $g_{\mathbf{X}}(\vec{x})$ is symmetric with respect to the lumen center along the curve's normal direction. In other words, the random variable $\mathbf{X}$ has a mean value equal to the lumen center. By the central limit theorem, the combination of (2) and (4) suggests that the principal curve is located at the lumencenter position. This is identical to the solution of the following sum of squared difference (SSD) metric-based optimization that minimizes the discrepancy between the observations and the intensity model centered at $\tilde{\mathbf{f}}(t)$, i.e.,

$$
\mathbf{f}(t)=\arg \min _{\tilde{\mathbf{f}}(t)} \sum_{\vec{x} \in \mathbb{X}_{t}}\left(I(\vec{x})-h_{t}(\|\vec{x}-\tilde{\mathbf{f}}(t)\|)\right)^{2} .
$$

This solution requires much less computational effort to calculate. Thus, hereafter, we use (5) to find a principal curve from a grayscale image.

\section{Algorithm For Finding Principal CuRveS}

The algorithm to find a principal curve from a grayscale image volume is summarized in a flow diagram (see Fig. 1). It is based on an efficient algorithm proposed in [18] known as the polygonal line algorithm. The algorithm output is a set of $k$ polygonal lines that minimizes the squared difference between the observed and model intensities at input data points (image voxels in our case). The output complexity is only $O(k)$, contrary to the other two algorithms [17], [19] that give output with equal complexity to the number of image voxels. A typical image volume usually consists of millions of voxels. Nonetheless, less than a hundred points is sufficient to model a smooth vessel centerline. Therefore, $k$ is much less than the number of voxels. This makes the polygonal line algorithm an appropriate method to extract lumen centers.

\section{A. Initialization}

The user gives an initial principal curve. This curve coarsely depicts a vessel of interest with two end vertices. The vertices are selected on planar reformatted images of an image volume. They have to be selected at the lumen center and are fixed (not optimized) throughout the algorithm execution to avoid undesired shortening or lengthening of the estimated vessel centerline. This also permits the user to gain a certain control over the output. If the end vertices are difficult to accurately locate from the reformatted images, a plausible position can be computed, as suggested in [20], by finding the zero crossing of two functions defined by the image gradients and the eigenvectors of the Hessian matrices, which denote the basis of a vessel cross-sectional plane, in a local neighborhood. Additional vertices inserted in between may be required if the vessel course significantly deviates from a straight line. The precise locating of these vertices is however not necessary unless they are anchors, i.e., locations where the principal curve must pass through. As such, the amount of user interaction is in proportion with the vascular

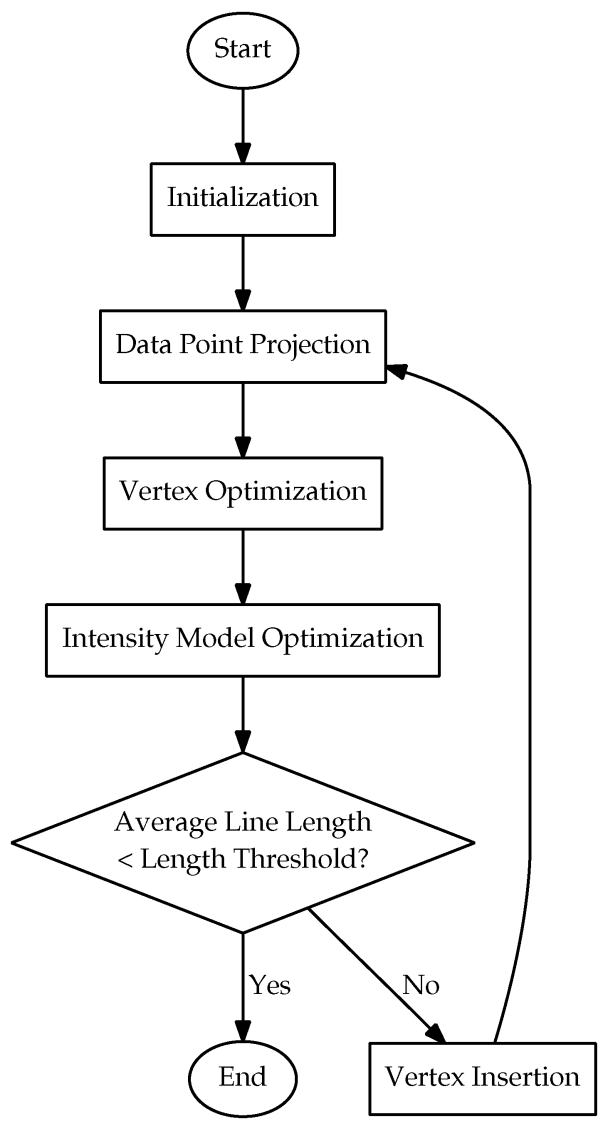

Fig. 1. Flow diagram of the polygonal line algorithm to find a principal curve.

complexity. In the given sequence of vertices, every adjacent pair defines a line. Each line has an associated intensity model that imitates the lumen-center peripheral image intensity. $\mathrm{Pa}-$ rameters of the model are set to their initial values in this step.

\section{B. Data Point Projection}

Following the initialization is a step that projects input data points onto the current principal curve $f$. Theoretically, this is to determine the projection index $\mathbf{f}^{-1}(\vec{x}), \forall \vec{x} \in \mathbb{X}$. However, knowing the exact index is not necessary, as we are going to update the principal curve in a discrete manner and the curve is nonparametrically represented as $k$ polygonal lines. As such, finding the curve location is equivalent to determining the end vertex locations of these lines. It is sufficient to project the data points onto these lines for evaluation of (5). Only points close to the current polygonal lines are considered. Other data points are too distant to have significant effect on determining the vertex locations. This prevents adjacent irrelevant structures from dictating the lumen-center extraction process. We thus project points that lay within the proximity to the curve [defined by parameter $a$ in (4)]. This volume, swept by the finite intensity model along a polygonal line, is cylindrical. We thus transform the projection step into finding the enclosure of input points in cylinders. There are $k$ cylinders of interest whose axes are the polygonal lines. Points inside a cylinder are projected onto the cylinder axis. If a point is covered by more than one cylinder, we project it onto the one with the shortest distance to the axis. The cylinder axes are oriented in 
a 3-D space depending on the principal curve vertex locations; computing the enclosure of points in an arbitrary oriented cylinder is not a trivial task. A computationally efficient option is to find an axis-aligned bounding box of the cylinder followed by an orthogonal range query with the uniform-level octree subdivision technique and, finally, an exhaustive search into the query result. This involves a series of range-searching problem. In this context, it is related to finding all the input points that lay inside the arbitrary oriented cylinders swept by the finite intensity model along the polygonal lines, which is known as range reporting. A state-of-the-art algorithm was developed in [21], namely, semialgebraic range searching. An arbitrary cylindrical range can be queried in near-linear time with linear range-searching data structure size. Notwithstanding the small data structure size, the way to resolve the query is not effective in this application. Our usual number of input points is of tens of millions; a million-order-of-magnitude query time is too much to report a few thousand or even hundred points. As such, we relax the problem to orthogonal range searching. Instead of resolving the difficult query in its tight range, we enlarge the query range to the cylinder's axis-aligned bounding box. Such a box range can be answered in polylogarithmic time with near-linear space-partitioning data structure (e.g., $k d$ tree, range tree, and octree [22]). This range relaxation saves up to five-orders-of-magnitude querying and reporting time. Since our input points are in latticed arrangement, we prefer the uniform-level octree as the space-partitioning data structure. Some reported points in this enlarged range are superfluous; we perform an exhaustive search ${ }^{1}$ in the query result to get rid of them. This approach gives a good tradeoff between the space and time complexity.

\section{Vertex Optimization}

Given the current principal curve, defined by a set of vertices, and $k$ sets of projected data points, we refine the curve by computing new vertex locations such that SSD between the observed and model intensities is further minimized. However, this refinement step is computationally difficult [18]. A suboptimal solution is obtained with a gradient descent method by optimizing SSD per vertex sequentially. The energy function to be minimized is a discrete version of (5), i.e.,

$$
E_{\text {data }}\left(V_{i}\right)=\frac{\sum_{j \in N\left(V_{i}\right)} \frac{1}{\left|\mathbb{L}_{j}\right|} \sum_{\vec{x} \in \mathbb{L}_{j}}\left(I(\vec{x})-h_{j}\left(\delta\left(\vec{x}, L_{j}\right)\right)\right)^{2}}{\left|N\left(V_{i}\right)\right|}
$$

where $V_{i}$ is the vertex of interest, $N(\cdot)$ returns indexes of the argument vertex's attached lines, $L_{j}$ denotes the $j$ th line, $\mathbb{L}_{j}$ is the projected data point set of the $j$ th line, $h_{j}(\cdot)$ is the intensity model of line $L_{j}$, and $\delta\left(\vec{x}, L_{j}\right)$ returns the shortest Euclidean distance of point $\vec{x}$ to the line segment $L_{j}$. The two denominators $\left|N\left(V_{i}\right)\right|$ and $\left|\mathbb{L}_{j}\right|$ are for normalization. We use a Butterworth-shaped function as the intensity model, i.e.,

$$
h_{j}(\|\vec{x}\|)=B+\frac{H}{1+(\|\vec{x}\| / R)^{2 n}}
$$

\footnotetext{
${ }^{1}$ The exhaustive search here means, for each returned point, discarding the point if it is outside the corresponding cylinder. This can be achieved by determining the shortest distance between the point and the axis of the cylinder.
}

where $B, H$, and $R$ denote background intensity, intensity amplitude at lumen center, and lumen half-width, respectively. They are all in $\mathbb{R}^{+}$. The lumen half-width is depicted by locations having intensity equal to half the center intensity amplitude over the background, i.e., $B+H / 2$. Parameter $n \in \mathbb{R}^{+}$ defines the rate of intensity drop at the half-width location. A large $n$ gives an abrupt drop. The model reduces to a rectangle function if $n$ is very large $(=50)$. A small $n(=1.5)$ gives a Gaussian-like function.

The principal curve is not parametric, and therefore, it may not be smooth if we optimize only $E_{\text {data }}(\cdot)$. An extra energy term is needed to regularize the curve, i.e.,

$$
E_{\text {strain }}\left(V_{i}\right)=\frac{\left(\left\|L_{j}\right\|^{2}+\left\|L_{j^{\prime}}\right\|^{2}\right)-\left(\left\|L_{j}^{0}\right\|^{2}+\left\|L_{j^{\prime}}^{0}\right\|^{2}\right)}{\left\|L_{j}^{0}\right\|^{2}+\left\|L_{j^{\prime}}^{0}\right\|^{2}}
$$

where $L_{j}$ and $L_{j^{\prime}}$ are the two attached lines of vertex $V_{i}$, and $L^{0}$. denotes a line before the optimization. Note that the formulation of (8) assumes that there are only two lines attached to any movable vertex. This energy term accounts for the relative strain induced by vertex dislocation due to an optimization. Strain, expressed as the difference in placement of parts in a system, is a measure of the degree of deformation. $E_{\text {strain }}(\cdot)$ returns a value in $\mathbb{R}$. It calculates the relative change in the attached lines' length, e.g., 0 denotes no change, 1 denotes $100 \%$ lengthening, and -0.2 means $20 \%$ shortening.

The overall energy function to be minimized becomes

$$
E\left(V_{i}\right)=E_{\text {data }}\left(V_{i}\right)+\lambda E_{\text {strain }}\left(V_{i}\right)
$$

where $\lambda \in \mathbb{R}^{+}$is a variable to control the degree of regularization. Analytic derivatives of the energy function are used to update the vertex locations. Neighboring vertex locations from the previous update are referred in the calculation. This is to mimic simultaneous updating of the locations. Thus, the vertex processing order is not important in this sequential gradient descent method. The vertex locations are iteratively updated until convergence, and a stopping criterion is implemented. Those vertices with location changes less than a threshold after a certain number of iterations are treated as inactive. The optimization step terminates until no active vertex or the maximum number of iterations is reached.

\section{Intensity Model Optimization}

Once we have updated the vertex locations, the optimal intensity model parameters of each polygonal line that best describe the projected data points' intensities are determined. This is performed by the following minimization:

$$
\left\{B^{*}, H^{*}, R^{*}, n^{*}\right\}=\arg \min _{\{B, H, R, n\}} \sum_{\vec{x} \in \mathbb{L}_{j}}\left(I(\vec{x})-h_{j}\left(\delta\left(\vec{x}, L_{j}\right)\right)\right)^{2} .
$$

A gradient descent method is used to calculate the optimal parameters. The parameters are optimized on a bounded space. Prior knowledge of the lumen cross-sectional intensity profile gives the parameters' boundaries. For instance, suppose the image intensity is normalized to $[0,1]$; we know that vessels 
are the brightest objects on a dark background having either a Gaussian-like or rectangular cross-sectional intensity profile, and we know the possible range of the lumen width and the intensity of the dimmest vessel. Thus, we have $0 \leq B \leq B_{\max }$, $H_{\min } \leq H \leq 1, R_{\min } \leq \mathrm{R} \leq R_{\max }$, and $1.5 \leq n \leq 50$, where $B_{\max }$ is the maximum background intensity on a lumen periphery, $H_{\min }$ is the dimmest lumen intensity, and $\left[R_{\min }, R_{\max }\right]$ defines the possible lumen width range.

\section{E. Vertex Insertion}

After the two optimization steps, a midvertex is inserted into a polygonal line that is long and has a large model intensity discrepancy from the observed data. The candidate line is the one that maximizes the following metric:

$$
L^{*}=\arg \max _{L_{j}}\left\{\left\|L_{j}\right\| \sum_{\vec{x} \in \mathbb{L}_{j}}\left(I(\vec{x})-h_{j}\left(\delta\left(\vec{x}, L_{j}\right)\right)\right)^{2}\right\} .
$$

We bisect this line and copy its intensity model parameters to the two new lines. The next iteration then commences with the data point projection step. The algorithm keeps iterating until the average line length is below a threshold. To speed up the execution, one can bisect multiple lines. Since there are more than one candidate lines, we use a priority queue on the metric to find them. Further speedup is possible if the next-iteration optimization is restricted to the newly inserted vertices and their immediate neighbors. The way we insert midvertices makes the final principal curve adaptive to the vasculature complexity; less vertices are used to depict a straight vessel segment, whereas more vertices are placed in a strongly bended segment. Such a principal curve is sufficient for a rough vessel delineation. However, a second stage of refinement is needed if a fine vessel centerline is required. For example, a straight line joining two vertices may be good enough to model a mildly zigzag segment. If the modeling of the fine detail is desired, more vertices should be used. To introduce more vertices, we re-execute the algorithm by inserting midvertices to lines with length greater than a threshold and optimizing only parameter $R$ in the intensity model optimization. To avoid sudden changes in the estimated lumen width, $R$ is optimized in between the minimum and maximum $R$ values among the neighboring lines. The regularization term weight $\lambda$ in (9) is multiplied by factor $m>1$ to compensate for the exponentially increasing degrees of freedom and to further constrain the principal curve smoothness in the second stage of refinement.

\section{Application to Vascular Network With Furcations}

Up to this point, we have introduced a method to extract lumen centers of a single vessel. However, it has a limited application in a clinical environment where a whole vascular network or a portion of it is of interest. Our nonparametric representation can be extended with a slight modification to handle lumen-center extraction of furcations because there is no need to maintain the order of vertices in such a representation. A set of vertices and an adjacency list allow representations of any branching configuration, even vascular network with loops, e.g., Circle of Willis in the cerebral circulation. The modification

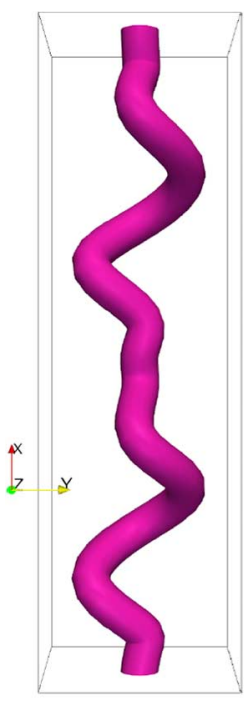

(a)

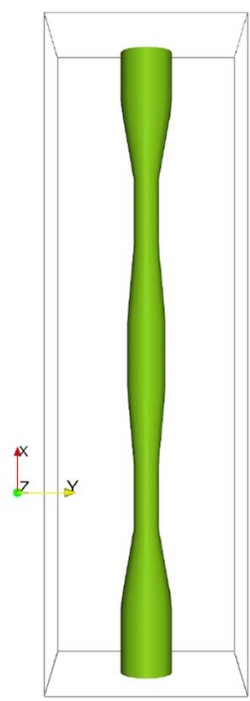

(b)

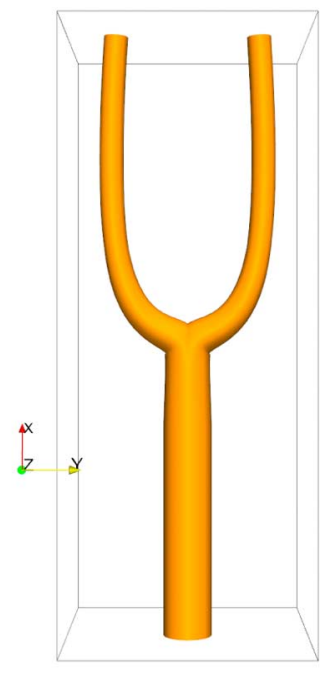

(c)
Fig. 2. Numerical models: (a) a curved tube, (b) a straight tube, and (c) a Y-shaped bifurcated tube. Visualization of these models is based upon a truncated cone methodology [5]. A surface model is generated as a triangle mesh from a sequence of tube centers and the associated radii.

is in the formulation of $E_{\text {strain }}(\cdot)$ given in (8). This formulation assumes that there are only two lines attached to any movable vertex. This assumption is violated in the case of furcation, e.g., at a bifurcation, three lines are attached to the branching vertex. To calculate the strain energy, we average the strain energies of all 2-combinations from the attached lines, e.g., there are $C_{2}^{4}=6$ strain energies to average in case of trifurcation; a vessel divides into three branches.

\section{VALIDATION AND DEMONSTRATION DESIGN}

The validation was conducted on three data pools, i.e., numerical phantoms, the BrainWeb [23], and the Rotterdam Coronary Artery [24] databases. The evaluation metrics, the initialization, and the setting of algorithm parameters for each data pool are described in this section. The demonstration details on two publicly available clinical data sets are also given.

\section{A. Simulated Data}

Three simulated data sets have been designed for studying robustness to intensity nonuniformality (INU), i.e., changes in image resolution, voxel anisotropy, and various levels of noise. We have also investigated the sensitivity to location variation of a user-given additional vertex in between the fixed end vertices. The first two data sets are images of a curved tube and a straight tube in a 3-D space of size $200 \times 60 \times 60$ arbitrary units (au); the last data set consists of a Y-shaped bifurcated tube image in a volume of $200 \times 80 \times 80$ au. All simulated data sets are of $1 \times 1 \times 1$ au voxel size and have intensity values 0 and 1 for voxels outside and inside the tubes, respectively. As shown in Fig. 2(a), the curved tube has a fixed width of 6 au. The straight tube [see Fig. 2(b)] starts with a width of 16 au, changes gradually to $8 \mathrm{au}$, widens to $12 \mathrm{au}$ at the volume center, and then narrows back to 8 au before it ends with a width of 16 au. The Y-shaped bifurcated tube, as presented in Fig. 2(c), aligns with the $x$-axis having an 8-au-wide base, a 6.5-au-wide junction, and 


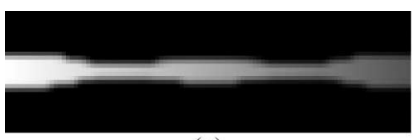

(a)

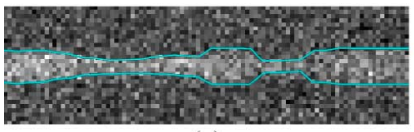

(c)

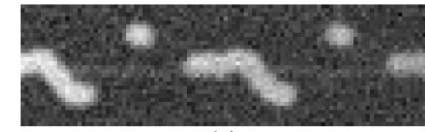

(e)

Fig. 3. Slices from the straight (str.)- and curved-tube data sets under various simulated imaging conditions.

two 4-au-wide branches. The lumen-center locations and widths were determined by using our method from the simulated data sets under various imaging conditions, i.e., three different levels of INU $(0 \%, 20 \%$, and $40 \%)$, four different resolutions (original, downsampled by a factor of 2 and 4 in all directions, and by a factor of 4 in the $z$-axis direction), and three noise levels (10, 5, and $2.5 \mathrm{~dB}$ ). INU was implemented as a multiplicative field with numbers ranging from $(1-y / 200)$ to $(1+y / 200)$ for $y \%$ INU. Fig. 3(a) shows a slice from a straight tube image with $40 \%$ INU. The noise level in decibels is defined as $10 \log _{10}\left(A / \sigma_{g}\right)$, where $A$ is the maximum image intensity and $\sigma_{g}$ is the zero-mean additive Gaussian noise standard deviation, e.g., if $A=1,5 \mathrm{~dB}$ implies $\sigma_{g}=0.32$ and $2.5 \mathrm{~dB}$ gives $\sigma_{g}=0.56$.

1) Evaluation Metrics: The accuracy of lumen-center extraction was quantified by the shortest Euclidean distances to the truth lumen centers of the curved tube. The principal curve was equidistantly sampled at a rate of $0.05 \mathrm{au}$. Then, we found the closest line segment of the truth curve defined by two adjacent lumen centers to each sample. The shortest Euclidean distance between the sample and the line segment was calculated. Lumen width estimation was quantitated using the straight tube. Output sensitivity to the additional vertex's location was studied in the bifurcated tube. Ten user-selected junction vertices were tested, giving ten sets of initial lumen centers. These vertices were picked from the proximity of the $\mathrm{Y}$-shaped junction having the farthest one at a distance of four times the junction width. The shortest Euclidean distances to one set of the centers from those in the remaining nine sets were recorded. Our method was evaluated with reference to a baseline condition, i.e., $20 \%$ INU, downsampled by a factor of (DF) 2 and $10 \mathrm{~dB}$. By altering a single factor (either INU, DF, or decibels) in the baseline condition, results under these different conditions were compared with that of the baseline.

2) Initialization and Parameter Settings: Initial principal curves were chosen to roughly follow the tube trajectories. Two end vertices defined the initial straight tube centerline, eight additional vertices along the course of the curved tube were inserted in between the two fixed end vertices for the initial curved centerline, and a single vertex was selected close to the junction of the $\mathrm{Y}$-shaped tube to initiate a bifurcated centerline. The intensity model parameters were initialized to the following values: $B=0, H=1, R=6 \mathrm{au}$, and

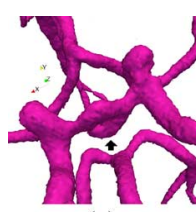

(a)

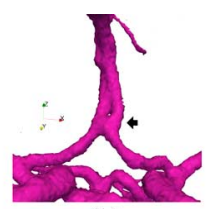

(b)

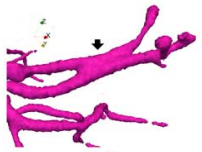

(c)

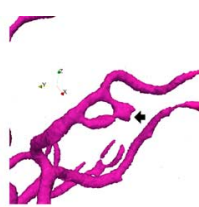

(d)
Fig. 4. (a) Spurious bulge at Circle of Willis in subject 06. (b) Merged abutting ACA: left and right ACAs in subject 04. (c) Merged MCA branches in subject 05. (d) Merged PCA branches in subject 45.

$n=4$. Settings of the algorithm parameters were $B_{\max }=1$, $H_{\min }=0.25, R_{\min }=2 \mathrm{au}, R_{\max }=9 \mathrm{au}$, the regularization term weight $\lambda=10^{-3}$, its multiplier in the second stage of refinement $m=5$, and the intensity model span $a=R+3 \Delta$, where $\Delta$ is the largest voxel dimension.

\section{B. BrainWeb Data}

To test our method on real vasculature that consists of varying-width vessels, bent vessels, and furcations, we generated 19 synthetic volumes based on the BrainWeb [23] vascular models. ${ }^{2}$ The size of each volume is $362 \times 434 \times 362$. The voxel spacing along the $x-, y$-, and $z$-axes is $0.5 \mathrm{~mm}$. The available binary vessel volumes were convolved with a Gaussian kernel of $3 \times 3 \times 3$ voxels $(\sigma=1)$ to mimic the point spread function of an imaging system and introduce partial volume effect due to a limited imaging resolution; the vessel boundaries were thus blurred. INU was introduced to the convolved volumes to simulate the variable B1-field of a radio-frequency coil. The inhomogeneity field A from the BrainWeb was employed. Zero-mean Gaussian noise was added. Test images are at $20 \%$ INU and $10 \mathrm{~dB}$. We focused on arteries because of their clinical relevance; although both arteries and veins are available, the carotid and vertebrobasilar systems are the objects of interest. The BrainWeb vascular volumes of normal subjects, however, are not perfect; the vascular structures provided are, in general, topologically or morphologically incorrect (i.e., with holes/cavities, handles, and bulges). There are spurious bulges at the Circle of Willis in several data sets. Very often, abutting vessels of the anterior cerebral artery (ACA), the middle cerebral artery (MCA), and the posterior cerebral artery (PCA) are merged. Examples are given in Fig. 4. These problematic structures were excluded in this paper.

1) Evaluation Metrics: We propose two ways of validation. One way is to voxelize the vascular models obtained from our algorithm (defined by lumen centers and widths) and quantify the discrepancy between the truth segmentations and the voxelized models. Voxels with their center located inside the domain spanned by the extracted lumen centers and their associated widths are included in the voxelized model. The Dice similarity coefficient (DSC) [25] was used to evaluate discrepancy. DSC ranges from 0 to 1 with a value greater than 0.7 indicating an excellent agreement between the two testing segmentations [26]. The other way is to measure the perpendicular distances from every vertex of the truth segmentation isosurface to our vascular models' circumference. We generated the isosurface with the marching cubes [27].

${ }^{2}$ Subject 52 was excluded from the 20 available BrainWeb vascular models due to the absence of major cerebral arteries. 
2) Initialization and Parameter Settings: End vertices of the initial principal curves were selected, which were located at the center of the lumens of interest. Typical locations are the root of internal carotid arteries (ICAs) and basilar artery [BA; or vertebral arteries (VAs), if any], the ends of distal MCA and PCA branches, and arteries that are proximal to the problematic structures. Furcation vertices were picked on an isosurface that facilitated interactive vasculature inspection as suggested in [16]. Those vertices were movable during the vertex optimization step. The adjacency list was compiled according to the observed vasculature or a vascular anatomical atlas. Since the solution is suboptimal, the initial curves have to be in the vicinity of the blood vessels. We adopted geodesic paths on the isosurface between the adjacent user-defined vertices as in [16] to refine the initialization. The end vertices were snapped to the isosurface before the geodesic path was computed. In most of the cases, there were too many vertices on the path and might cause loops within the vessels or inside nearby irrelevant structures. A greedy algorithm was used to simplify the geodesic path. Starting from a path end vertex, we walked through the path on a vertex-by-vertex basis and checked if the traveled path could be approximated by a straight line joining the end vertex and the last visited vertex. The appropriateness of such approximation was evaluated by the distance from all the intermediate path vertices to this straight line. If it was found inappropriate (if the maximum distance was greater than half of the associated width of the original polygonal line, i.e., the line defined by the user-given vertices), the previously tested straight line was used to approximate the transverse portion. The last transverse vertex was then treated as an end vertex, and the aforementioned process was reiterated until the other end vertex of the geodesic path was reached. Settings of the algorithm parameters were identical to those for the synthetic data, except for the following. $R$ that denotes the lumen half-width was set to the reference half-width of the target vessel segment, i.e., $3 \mathrm{~mm}$ for ICA; $2 \mathrm{~mm}$ for ACA, MCA, BA, and VA; and $1 \mathrm{~mm}$ for distal MCA branches, PCA, posterior communicating artery (PCoA), superior cerebellar artery (SCA), and anterior inferior cerebellar artery. $R_{\min }=0.5 \mathrm{~mm}, R_{\max }=3.5 \mathrm{~mm}$, and $m=10$.

\section{Rotterdam Coronary Artery Data}

The third data pool is obtained from the Rotterdam Coronary Artery Algorithm Evaluation Framework [24], which provides a large set of real clinical data for validation and is a standardized framework for the evaluation of the coronary computed tomography angiography (CTA) lumen-center extraction algorithms. This opens up opportunities for comparison between our method and other state-of-the-art algorithms (currently, 13 methods have been evaluated within the framework). Well-defined evaluation metrics are described, and reference standards of the coronary lumen centers are available. ${ }^{3}$ This data pool consists of 32 CTA data sets of various image quality and levels of arterial calcification [28].

The vessels of interest are four coronary arteries, viz., the right coronary artery (RCA), the left anterior descending artery

\footnotetext{
${ }^{3}$ Only the reference standards of the eight training data sets are publicly available; the other 24 sets are not provided to guarantee a unified evaluation and comparable results.
}

(LAD), the left circumflex artery (LCX), and one large side branch (LSB) of the main coronary arteries. In total, there are $32 \times 4=128$ sets of lumen centers to be extracted and evaluated. We followed the two preprocessing steps-in-plane downsampling and intensity clamping - stated in [29] for efficient computing, lower memory consumption, and exclusion of irrelevant structures. The coordinates of the extracted centers were submitted to the organizer for evaluation; therefore, only the lumen centers of our algorithmic outputs were validated.

1) Evaluation Metrics: The Rotterdam Coronary Artery Algorithm Evaluation Framework provides three overlap measures, i.e., overlap (OV), overlap until first error (OF), and overlap with the clinically relevant part of the vessel (OT), to quantify the ability to extract lumen centers, and a distance measure, i.e., average distance inside vessel (AI), to determine the extraction accuracy. A $0-100$ score is calculated per measure w.r.t. the performance of the observers; 50 points implies a similar performance. The detailed description of the measures can be found in [28]. In this paper, we are particularly interested in the OT and AI measures because we want to study our algorithm's capability of extracting the clinically relevant part of coronary arteries and its accuracy of those correctly extracted portions.

2) Initialization and Parameter Settings: The end vertices of the initial principal curves were the start point and the endpoint provided by the evaluation framework. To reduce the time spent on browsing an image volume for initialization, the Dijkstra algorithm was employed to obtain a simple lumen-center extraction between the end vertices and any additional anchors in between. We used an algorithm similar to the one proposed in [13], except that we used a single scale $(=0.75 \mathrm{~mm})$ to find the centerline from the image volume. The eight-neighborhood system was used for fast processing. The obtained centerline was usually composed of many vertices (the number of vertices is equal to the number of voxels that the centerline passes through). We employed the Douglas-Peucker algorithm [30] to simplify the centerline to the one that has tens of vertices. LAD, LCX, and the chosen LSB of the main coronary arteries share a common course proximal to the aortic ostium in most of the data sets. We initialized the principal curves to reflect this without duplicating the modeling of the common course [see Fig. 5(a)]. Furcation vertices were therefore introduced. End vertices and anchor points are fixed, while furcation vertices and those left-out vertices inserted by the Dijkstra algorithm are movable. This can be automatically configured via investigating the number of lines attached to a vertex of the initial principal curves prior to a simple extraction step, i.e., the end vertex has one line attached, the anchor has two, and the furcation vertex has $\geq 3$. Parameter settings were identical to BrainWeb, except parameters, i.e., $R$ was set to $0.75 \mathrm{~mm}, H$ to $0.3, R_{\min }$ to $0.75 \mathrm{~mm}$, and $R_{\max }$ to $3 \mathrm{~mm}$.

\section{Clinical Data}

We tested our algorithm on two publicly available 4 data sets, i.e., a 3-D rotational angiography (3-D RA) of the right half of a cerebral circulation $(256 \times 256 \times 256$ voxels $)$ in isotropic

\footnotetext{
${ }^{4}$ The two data sets are available at http://www.gris.uni-tuebingen.de/edu/ areas/scivis/volren/datasets/datasets.html.
} 


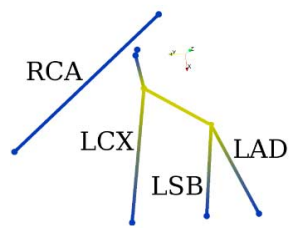

(a)

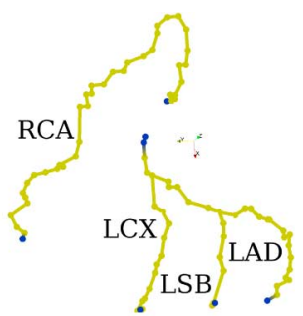

(b)
Fig. 5. Initial principal curves to extract RCA, LAD, LCX, and LSB of the coronary arteries from Data set 1 in the Rotterdam Coronary Artery database. (a) The user-given initial principal curves. All six end vertices are from the framework; three additional points are provided by the user, i.e., an anchor and two furcation points. The common arterial courses shared by LAD, LCX, and LSB are modeled by a single sets of line segments. End vertices and the anchor points are in blue (dark gray) and are fixed. End vertices are the start point and the endpoint provided by the Rotterdam Coronary Artery Algorithm Evaluation Framework. The anchor is in the proximity of the LCA root. Furcation vertices are in green (light gray) and are movable. They are the branching positions of LAD and LCX from LCA and of LSB. (b) The initial curves after the execution of the Dijkstra and Douglas-Peucker algorithms. Tens of vertices were inserted, and the curves roughly follow the coronary artery courses.

voxels $\left(1 \times 1 \times 1 \mathrm{~mm}^{3}\right)$ and a computed tomography $(\mathrm{CT})$ of the abdomen and the pelvis $(512 \times 512 \times 174$ voxels $)$ in anisotropic voxels $\left(0.8398 \times 0.8398 \times 3.2 \mathrm{~mm}^{3}\right)$. An aneurysm is present in the 3-D RA volume. The CT data set contains a stent graft in the abdominal aorta (AA). Voxel intensities were linearly rescaled to values between 0 and 1 , such that blood vessel lumens have intensity close to 1 , whereas the background value is close to 0 in the rescaled volumes. An additional step is needed in the CT scan to eliminate heterogeneous background and bright irrelevant structures, and the implementation is automatic. The intensity of the bone and the stent was set to that of muscle tissue. Any tissue having lower intensity than that of muscle tissue was assigned to have muscle intensity. Numerically, we linearly mapped CT intensity between 1000 and $1300 \mathrm{HU}$ to 0 and 1 . The intensity outside that range was clamped to 0 . We also downsampled the CT volume by a factor of 4 in the in-plane dimensions for fast processing. Settings of the algorithm parameters were identical to those for BrainWeb, except that $R$ was initialized to the reference half-width of the target vessel segment and $R_{\min }$ and $R_{\max }$ were set to numbers that can cover a wider width range of the lumens of interest. For 3-D RA data set, $R$ ranges from 1 to $5 \mathrm{~mm}, R_{\min }=0.5 \mathrm{~mm}$, $R_{\max }=6 \mathrm{~mm}$, and $m=100$. For the CT data set, we set $R$ to values between 3 to $15 \mathrm{~mm}, R_{\min }=1.5 \mathrm{~mm}, R_{\max }=17 \mathrm{~mm}$, and $m=5$.

\section{RESUlts}

\section{A. Simulated Data}

Percentiles of the shortest Euclidean distances to the truth locations of the curved tube lumen centers are plotted in Fig. 6. Our method outperformed the method proposed by Frangi et al. (the Hessian filter scale was set to the lumen width of 6 au; a cardinal spline [31] was used to model the vessel axis) under the baseline condition and gave results in subvoxel accuracy. Our maximum error is less than one-third of the voxel diagonal. This is in sharp contrast to the maximum error, which is $120 \%$ of the voxel diagonal, obtained by using the method by Frangi et al.
Percentiles of Shortest Euclidean Distances to the Truth Locations of Curved Tube Lumen Centers

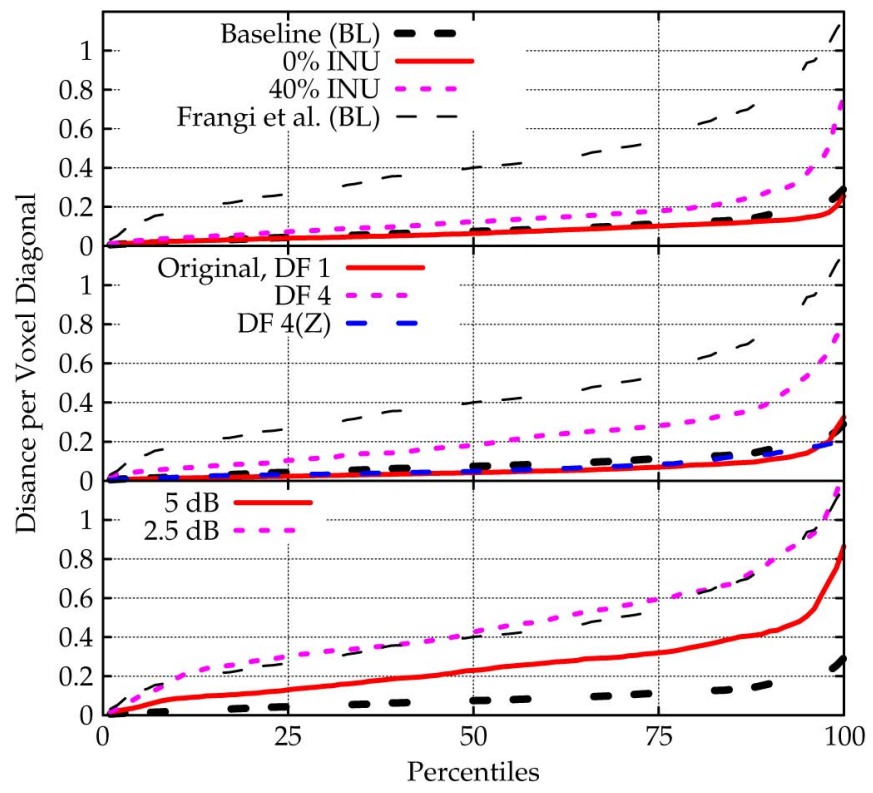

Fig. 6. Percentiles of the shortest Euclidean distances to the truth locations of curved tube lumen centers. Tests were conducted under various imaging conditions: three INU levels $(0 \%, 20 \%$, and $40 \%$ ), four different resolutions (original, downsampled by a factor of (DF) 2 and 4 in all directions, and by a factor of 4 in $z$-axis direction), and three noise levels $(10,5$, and $2.5 \mathrm{~dB})$. The baseline (BL) condition is $20 \%$ INU, DF 2 , and $10 \mathrm{~dB}$. A single factor (INU, DF, or decibels) in the baseline condition is altered and tested. Results were obtained under individual tested condition and were compared with that of the baseline. The method by Frangi etal was tested under the baseline condition.

Various imaging conditions do not adversely affect our subvoxel accuracy. All our shortest Euclidean distances per voxel diagonal are well below 1.0 except under severe noise $(2.5 \mathrm{~dB})$. Our method is particularly resistant to the intensity variation along the tube course. The shortest Euclidean distances do not surge under $40 \%$ INU (see the top plot of Fig. 6). Voxel anisotropy poses no difficulty for our method. The curved lumen centerline extracted is as good as the one obtained from the image at the original resolution (see line DF 4 ( $z$-axis) in the midplot of Fig. 6).

Fig. 3(d) shows a slice from the 2.5-dB data set in which our method fails to give subvoxel accuracy. It is indeed very challenging for any algorithm to extract curved centerline from such a noisy environment. Interestingly, our result is comparable with the one obtained by the algorithm by Frangi et al. under a less noisy condition [the baseline condition; see Fig. 3(e) for a slice under that condition]. Further investigation suggested that this was due to a shift in the maximum vesselness measure toward the curvature center. The centerline that followed the trajectory of the maximum measures thus drifted away from the true lumen centers at high curvature regions. This problem is prominent for a strongly bent lumen whose curvature radius is of the lumen width order [16]. To get a satisfactory result from curved tube data set, the initialization of the method by Frangi et al. needed 18 additional vertices in between the two fixed end vertices. Locations of these vertices are crucial; more vertices have to be placed in the high curvature portion of the tube. The centerline degree of freedom is insufficient for the model 


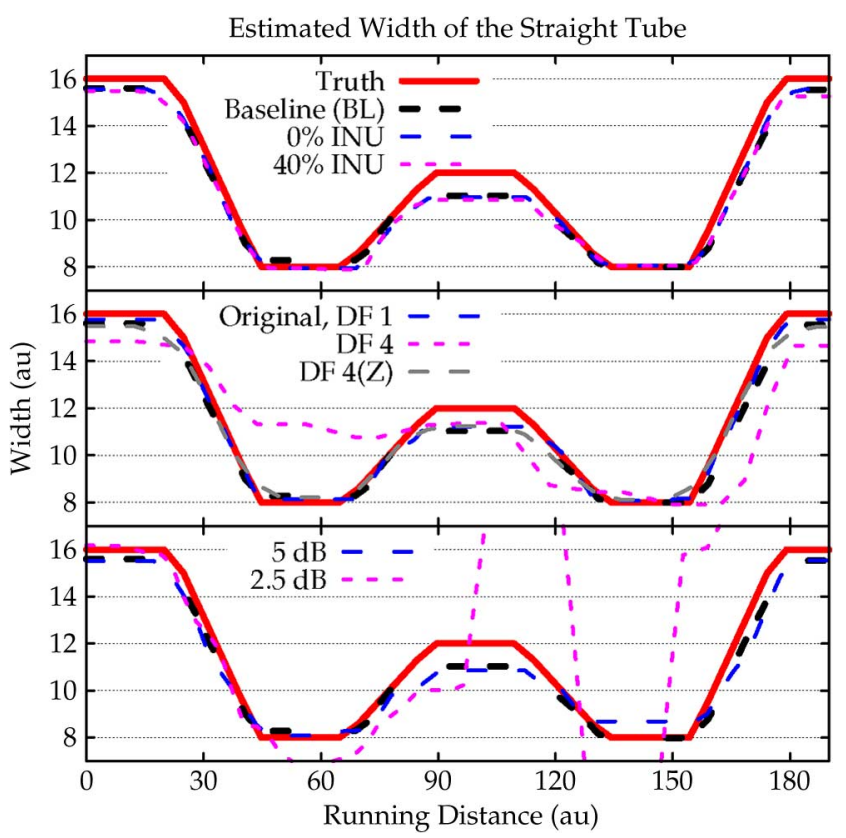

Fig. 7. Estimated width of the straight tube against the running distance along the tube course. Tests were conducted under various imaging conditions, and results were compared with the one obtained under the baseline condition. For details of the baseline (BL) condition and other imaging conditions, see the caption of Fig. 6.

to depict the curvy vasculature otherwise. On the contrary, our method is adaptive to vasculature complexity. More movable vertices can be automatically added to model the curvy vessel segment.

We obtained similar findings from the straight tube data set. Our estimated lumen widths were subvoxel accurate under various imaging conditions. Intensity variation and voxel anisotropy do no harm our method performance. Lines presented in Fig. 7, which shows the estimated widths under conditions $40 \%$ INU, DF 4 ( $z$-axis), and $5 \mathrm{~dB}$, are aligned with that of the baseline. Width estimation errors are less than 1 au in all these cases. Although significant deviation is noted at the line DF 4 of the midplot, the errors are well under the voxel size 4 au. Fig. 3(b) shows a slice of the DF 4 data set with the approximated tube outline overlaid. Subvoxel accuracy is not hard to perceive. The $2.5-\mathrm{dB}$ data set remains very challenging. Conspicuous errors are shown in the bottom plot of Fig. 7. A study examining the data set and the estimated tube (Fig. 3(c) presents a slice and the tube outline) suggests that the results are satisfactory.

Percentiles of distances between lumen centers extracted from the $Y$-shaped bifurcated tube with different user-selected junction vertices are presented in Fig. 8. The lumen centers were extracted from a data set under the baseline condition. Our method is fairly insensitive to the user-selected location. The lumen centers obtained with different initial curves are nearly identical. Lines in the plot are coincided. The maximum difference and $95 \%$ of the differences are less than one third and one twentieth of the voxel diagonal, i.e., 1.15 and 0.18 au, respectively. Fig. 9 shows the initial and final principal curves together with the Y-shaped bifurcated tube. Visual inspection of the extracted bifurcated centerlines confirms our claim.

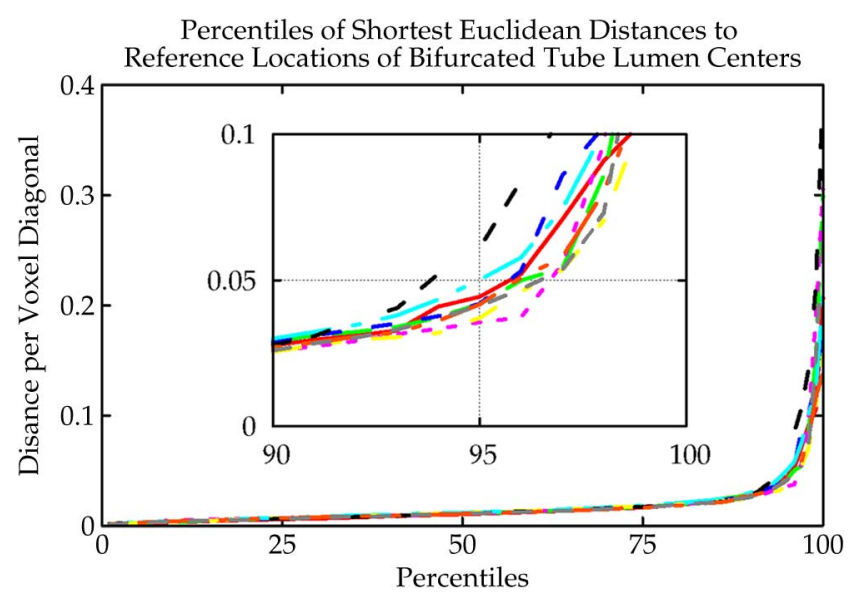

Fig. 8. Percentiles of the shortest Euclidean distances between lumen centers extracted from the Y-shaped bifurcated tube with different user-selected junction vertices. Ten user-selected junction vertices were tested, and thus, ten sets of lumen centers were extracted. The junction vertices were picked from the proximity of the Y-shaped junction. We treated one set of the centers as the reference; distances from those in the rest nine sets were evaluated. The baseline imaging condition (20\% INU, DF 2, and $10 \mathrm{~dB}$ ) was used.

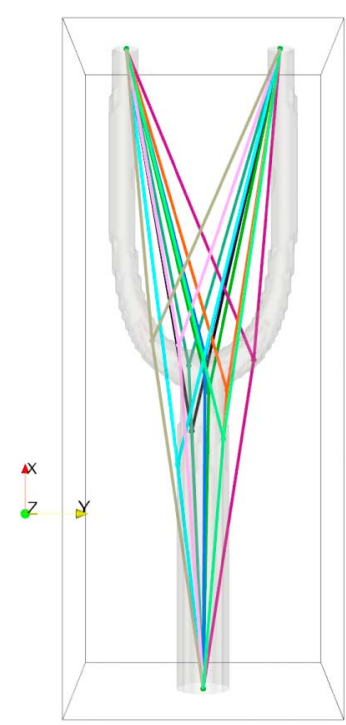

(a)

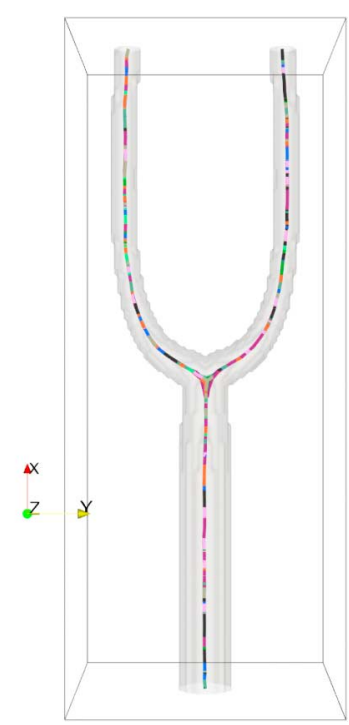

(b)
Fig. 9. Ten (a) initial and (b) final principal curves of the Y-shaped bifurcated tube under the baseline condition ( $40 \%$ INU, DF 2 , and $10 \mathrm{~dB})$. The final curves depict the tube lumen centers.

\section{B. BrainWeb Data}

Although our vascular model assumes a circular cross section, its voxelized volume is in excellent agreement with the truth segmentation. As plotted in Fig. 10(a), DSC values of the 19 tested BrainWeb subjects are all greater than 0.9, far above the benchmark of 0.7. Furthermore, our models provide satisfactory surface representation of the truth segmentations. The vast majority of the perpendicular distances from the isosurface vertices to the models' circumference are at the subvoxel level. As shown in the box-percentile plot [32] (displays perpendicular distances up to $0.60 \mathrm{~mm}$ ) in Fig. 10(b), all the 95 percentiles are less than $0.60 \mathrm{~mm}$, which is far smaller than the voxel diagonal length (vd) of $0.87 \mathrm{~mm}$. Average values of the 50, 95, and 99 (not shown in the plot) percentiles are $0.14,0.49$, and $0.77 \mathrm{vd}$, respectively. The average maximum deviation from the isosurfaces is 


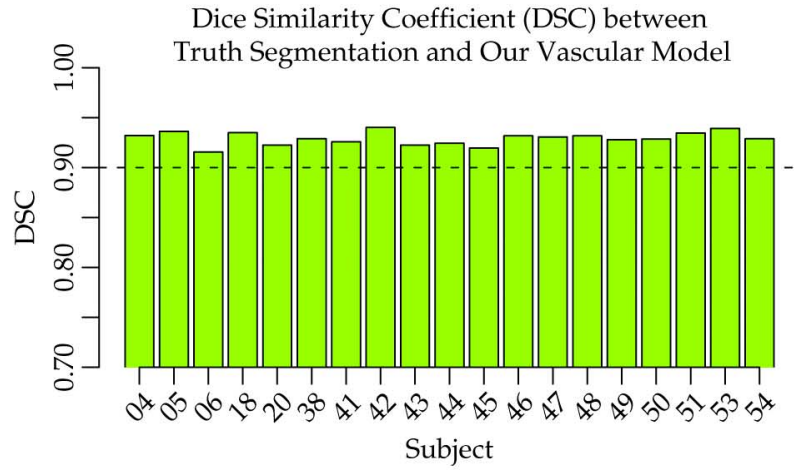

(a)
Perpendicular Distances from Iso-surface Vertices to Our Vascular Models' Circumference

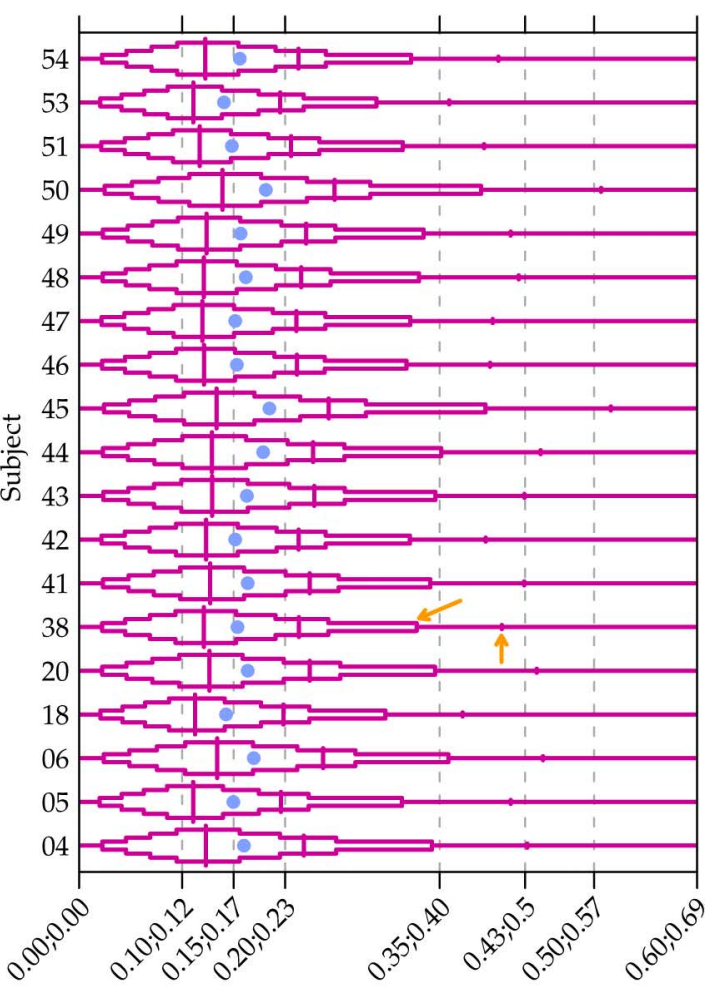

Perpendicular Distance (mm;vd)

(b)

Fig. 10. (a) DSC between the truth segmentations and our vascular models of the 19 tested BrainWeb vascular phantoms. (b) Box-percentile plot of the perpendicular distances from the isosurface vertices to our vascular models' circumference in the 19 test BrainWeb data sets. Distances are shown in both millimeter and per voxel diagonal (vd) units. The width of the boxes is discretized with 5\% increments. The perpendicular distance mean of each subject is highlighted by the light (blue) dot. Four vertical marks across the boxes show 50, 75, 90, and 95 percentiles. The 90 and 95 percentiles' marks of subject 38 are pointed at by the arrows for better illustration.

$\approx 2 \mathrm{vd}$ (not shown in the plot); nonetheless, large deviations occupy only less than $1 \%$ of the total models' circumference.

\section{Comparison With a State-of-the-Art Method}

We have compared our method with the algorithm by Frangi et al. [16] on two BrainWeb data sets. Our focus is to evaluate the performance of the lumen-center extraction. Therefore, we only examined their central vessel axis model in the experiments. Scales of the Hessian filter spanned the possible lumen width in the data sets, i.e., $1-5 \mathrm{~mm}$. Five scales were used and selected according to a scale selection criterion [33]. We employed a cardinal spline [31] to model the vessel axis. In this comparison, potential pitfalls of the algorithm by Frangi et al. in extracting lumen centers at strongly bent vessel segments and branching portion were revealed. If there are not enough control points given, it can fail to track lumen centers at even a slightly bent segment. These pitfalls are illustrated in Figs. 11 and 12. Fig. 11(a) and (b) show the horizontal (M1) segment of MCA in subject 54 together with the extracted lumen centers. Dark (purple) lines are obtained from our method, and light (orange) lines are from the method of Frangi et al. Three times more control points were used to get the light line in Fig. 11(b), as compared with the one presented in Fig. 11(a). Both methods were applied on the grayscale synthetic images with the same initial curve as input. It is found that the number of control points has a large effect on the parametric approach. Accuracy can deteriorate even in modeling slightly bent segments if there is an insufficient number of control points. Due to the restricted degrees of freedom, the spline in Fig. 11(a) fails to track the M1 segment axis. Severe deviation is observable in the distal portion. On the contrary, our lumen centers are satisfactory. They fall in the middle of the segmentation isosurface silhouette in the rendered image. Fig. 11(b) illustrates that, if more control points are added to the spline, the accuracy can be improved in this particular case.

Another example shown in Fig. 11(c) is the BA of subject 49. A large deviation is found in the proximal region because of too few spline control points. As presented in Fig. 11(d), using more control points can give excellent results. Nevertheless, the parametric approach can become unstable when more iterations are allowed in the energy optimization. The spline can develop a loop within the vessel, as shown in Fig. 11(e). One possible reason is that local sharp turns are not effectively inhibited with the integration-based energy functional. Energy is increased due to large first- and second-order derivatives at these turns if the spline is long. In addition, the spline is allowed to go through multiple times the region where the Hessian filter responses are high. The gradient descent optimization method can easily get 


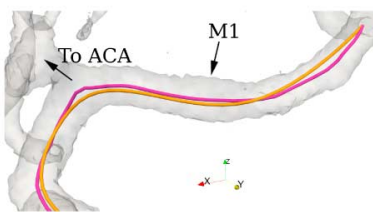

(a)

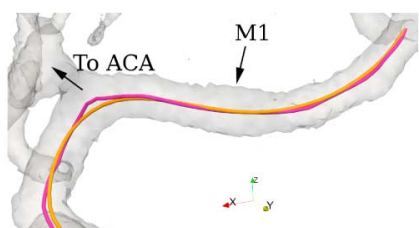

(b)

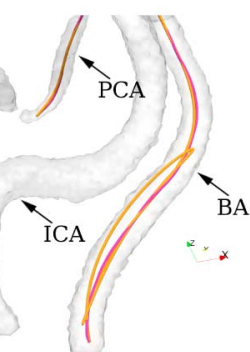

(e)

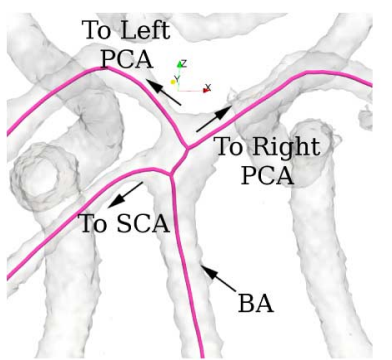

(a)

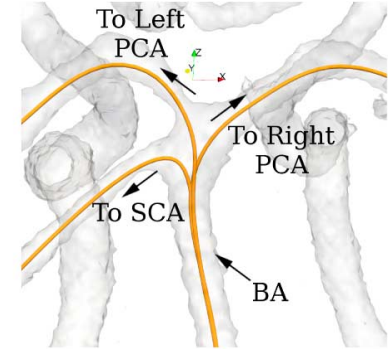

(b)
Fig. 12. Lumen centers extracted with our algorithm and algorithm of Frangi etal [16] on a BrainWeb data set: subject 49. The furcation at the terminal of BA is shown. The branches to left SCA and the PCAs are present. These vessel segments are highlighted and named. Dark (purple) lines are obtained from our algorithm, and light (orange) lines are from the method of Frangi etal The transparent segmentation isosurfaces are for visualization only. They were not the algorithm input. (c)

(d)

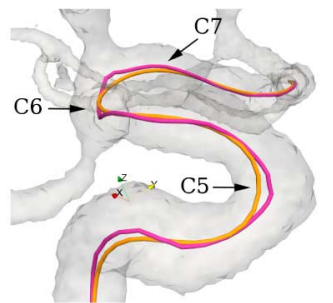

(g)
Fig. 11. Lumen centers extracted with our algorithm and the algorithm of Frangi etal [16] on two BrainWeb data sets: subjects 49 and 54. The first row shows the horizontal (M1) segment of MCA in subject 54, the BA of subject 49 is shown in the middle row, the last row shows the C5, C6, and C7 ICA segments of subject 54. Dark (purple) lines are obtained from our algorithm, and light (orange) lines are from the method of Frangi etal (a), (c), and (f) show the algorithm outputs of Frangi etal with too few spline control points. (b), (d), and (g) show the outputs with more control points. (e) presents the output with more iterations in the energy optimization of algorithm of Frangi etal. The transparent segmentation isosurfaces are for visualization only. They were not the algorithm input. A few major vessels are highlighted and named in the figures.

caught at a local minimum in such high-dimensional solution space. Setting higher stretch and bending energy weights in this case can help, but we have also noticed undesired effects on other parts because these weights affect the results globally.

At the C5, C6, and C7 ICA segments of subject 54, more spline control points do not give a satisfactory result, as demonstrated in Fig. 11(g). Investigating the multiscale Hessian filter responses suggests that it is a drawback to convolve images with filters. Filter responses are biased to curvature center at a strongly bent lumen with curvature radius comparable with its width [16]. This explains the spline drift toward the inner side of a curved segment as exemplified in Figs. 11(f) and (g). A similar problem is also observed at branches as depicted in Figs. 11(a), (b), and 12(b), as compared with our results shown in the dark (purple) lines, and in Fig. 12(a), parametric splines cannot track the lumen centers at furcations. The splines are biased toward the boundary facing the curvature center.

Furthermore, modeling branching vasculature with the method by Frangi et al. is not as elegant as ours. Their method tracks the incidence artery axis with multiple splines, and the lumen centers of segment at the junction may not be well modeled. In contrast with our results presented in Fig. 12(a), the extracted lumen centers from the method of Frangi et al. given in Fig. 12(b) start to diverge at the proximal part of the BA terminal junction; the very short BA segment following the branch to SCA is not appropriately modeled.

\section{Rotterdam Coronary Artery Data}

The evaluation results issued by the organizer are tabulated in Tables I-VI. They are also available online 5 for the performance comparison against other evaluated state-of-the-art methods. The average overlap measure OT (see Tables I and IV) among all the data sets is $>96 \%$ with a score $\gg 50$. This indicates our algorithm has a capability of providing a close-to-complete extraction of the (clinically relevant) coronary arteries. Subvoxel accuracy is also observable. The average distance inside vessel AI (see Tables II and V) is $\leq 0.31 \mathrm{~mm}$; this is $\leq 30 \%$ of the intrinsic voxel diagonal length 6 (VD). However, it is noted that the minimum overlap measures, particularly OF, are low, and the maximum average distance measure is high (see Tables III and VI). An investigation into the available reference standards (of the eight training data sets 0-7) suggests three reasons for those statistics: 1) The observers were asked to trace lumen centers of several arterial segments as a single artery. The identified centers of the inlet segment near a junction are biased toward the inner side of the curved course. On the contrary, our method considers the arterial network (inlet segment and its outlets) as a whole in the extraction process. This favors a successful lumen-center extraction in the individual segment (see Fig. 13). 2) In a few cases, our algorithm did not correctly extract the lumen centers of distal, low contrast, and narrow coronary arteries. Slight deviations from the reference standards are noticeable, but they are of relatively low clinical relevance. A solution to this problem is to click more anchor points along the course in the initialization. We intentionally did this in a few data sets and found that the overlap measures surge to $100 \%$. 3) Some calcium voxels were left out in the

\footnotetext{
${ }^{5}$ The evaluation results are published at http://coronary.bigr.nl/results/results. php.

${ }^{6} \mathrm{As}$ pointed out in [29], there is no power in the frequencies $>\pi / 2$ of the image spectra. This implies that the intrinsic resolution of the in-plane images is $256 \times 256$ rather than $512 \times 512$. Thus the downsampling by a factor of 2 does not hurt the accuracy.
} 
TABLE I

Average Overlap and Number of Anchor Points Per Testing Data Set. Please Refer to Section V-C-1 for the Meaning of Evaluation Metrics. The Column Rank Lists the Ranking (THE Least, 1.00, AND the Better) Of Our Method AMONGST ALL THE 14 EVALUated STATE-OF-THE-ART METHODS

\begin{tabular}{|c|c|c|c|c|c|c|c|c|c|c|c|c|c|c|c|}
\hline \multirow{2}{*}{$\begin{array}{c}\text { D } \\
\text { nr. }\end{array}$} & \multicolumn{3}{|c|}{ OV } & \multicolumn{3}{|c|}{ OF } & \multicolumn{3}{|c|}{ OT } & \multirow{2}{*}{$\begin{array}{l}\text { Avg. } \\
\text { rank }\end{array}$} & \multicolumn{5}{|c|}{ Anchor pts } \\
\hline & $\%$ & score & rank & $\%$ & score & rank & $\%$ & score & rank & & RCA & LAD & $\mathbf{L C X}$ & LSB & Total \\
\hline 0 & 100.0 & 100.0 & 1.00 & 100.0 & 100.0 & 1.00 & 100.0 & 100.0 & 1.00 & 1.00 & 6 & 3 & 2 & 3 & 9 \\
\hline 1 & 97.4 & 86.2 & 2.50 & 96.9 & 85.9 & 1.75 & 97.4 & 86.2 & 2.50 & 2.25 & 0 & 3 & 2 & 3 & 3 \\
\hline 2 & 100.0 & 100.0 & 1.00 & 100.0 & 100.0 & 1.00 & 100.0 & 100.0 & 1.00 & 1.00 & 0 & 8 & 2 & 4 & 9 \\
\hline 3 & 96.2 & 89.6 & 2.50 & 83.3 & 77.4 & 2.75 & 96.2 & 88.9 & 2.50 & 2.58 & 0 & 16 & 2 & 3 & 16 \\
\hline 4 & 94.7 & 72.9 & 3.75 & 76.0 & 63.2 & 4.50 & 98.6 & 74.6 & 3.25 & 3.85 & 1 & 5 & 10 & 2 & 15 \\
\hline 5 & 96.7 & 78.4 & 2.75 & 71.0 & 60.5 & 4.75 & 96.7 & 73.4 & 3.50 & 3.67 & 0 & 3 & 1 & 4 & 4 \\
\hline 6 & 98.3 & 74.0 & 4.00 & 95.1 & 71.9 & 4.00 & 99.5 & 87.0 & 3.50 & 3.83 & 5 & 3 & 2 & 3 & 8 \\
\hline 7 & 97.2 & 74.3 & 2.25 & 94.6 & 72.6 & 1.25 & 98.6 & 86.9 & 1.25 & 1.60 & 6 & 14 & 7 & 9 & 31 \\
\hline Avg. & 97.6 & 84.4 & 2.47 & 89.6 & 78.9 & 2.62 & 98.4 & 87.1 & 2.31 & 2.47 & 2.3 & 6.9 & 3.5 & 3.9 & 11.9 \\
\hline
\end{tabular}

TABLE II

AVERAge ACCuracy and VoXel Diagonal Length PER TESTING Data SeT

\begin{tabular}{|c|c|c|c|c|c|}
\hline D & \multicolumn{3}{|c|}{$\mathbf{A I}$} & \multirow{2}{*}{$\begin{array}{l}\text { Avg. } \\
\text { rank }\end{array}$} & \multirow{2}{*}{$\begin{array}{l}\mathrm{VD} \\
\mathrm{mm}\end{array}$} \\
\hline nr. & $\mathrm{mm}$ & score & rank & & \\
\hline 0 & 0.37 & 39.6 & 3.75 & 3.75 & 1.10 \\
\hline 1 & 37 & 33.2 & 5.50 & 5.50 & 1.10 \\
\hline 2 & 15 & 40.3 & 3.75 & 3.75 & 1.03 \\
\hline 3 & 3 & 40.7 & 5.25 & 5.25 & 1.12 \\
\hline 4 & 27 & 34.6 & 5.25 & 5.25 & 0.98 \\
\hline 5 & 36 & 40.1 & 5.25 & 5.25 & 1.00 \\
\hline 6 & 28 & 33.4 & 4.50 & 4.50 & 0.99 \\
\hline 7 & 0.27 & 36.2 & 4.25 & 4.25 & 0.91 \\
\hline Avg & 0.31 & 37.3 & 4.69 & 4.69 & 1.03 \\
\hline
\end{tabular}

TABLE III

Summary OF THE Testing SeT (DATA SeT 8-31)

\begin{tabular}{|c|c|c|c|c|c|c|c|c|c|}
\hline & \multicolumn{3}{|c|}{$\% / \mathrm{mm}$} & \multicolumn{3}{|c|}{ score } & \multicolumn{3}{|c|}{ rank } \\
\hline & $\min$. & $\max$. & avg. & $\min$. & max. & avg. & min. & $\max$. & avg. \\
\hline OV & $83.3 \%$ & $100.0 \%$ & $97.6 \%$ & 42.4 & 100.0 & 84.4 & 1 & 9 & 2.47 \\
\hline OF & $9.9 \%$ & $100.0 \%$ & $89.6 \%$ & 5.0 & 100.0 & 78.9 & 1 & 10 & 2.62 \\
\hline OT & $87.7 \%$ & $100.0 \%$ & $98.4 \%$ & 44.6 & 100.0 & 87.1 & 1 & 7 & 2.31 \\
\hline $\mathrm{AI}$ & $0.17 \mathrm{~mm}$ & $0.42 \mathrm{~mm}$ & $0.31 \mathrm{~mm}$ & 25.3 & 50.6 & 37.3 & 2 & 8 & 4.69 \\
\hline Total & & & & & & & 1 & 10 & 3.58 \\
\hline
\end{tabular}

intensity clamping, and our algorithm confuses these bright spots with lumen centers. An extra anchor point at the desired centerline can correct this. Of those testing data sets in which our method gave low minimum overlap measures and a high maximum AI, we found that either their image quality is poor or the calcification is severe.

We present the number of anchor points manually clicked per artery and per data set in Tables I and IV. The total number of anchor points is less than the sum of the anchor number per artery due to the common arterial course shared among LAD, LCX, and the selected LSB. Data set 1 is the representative of such a configuration [see Fig. 5(a)]. The start pont and the endpoint from the evaluation framework are good enough to extract RCA. An anchor point at the root of the left coronary artery (LCA) is oftentimes needed because the given start point of the other three vessels of interest is laid inside the aorta, and our method may falsely extract lumen centers inside the aorta. In some cases, additional anchors are necessary to constrain the algorithm not to extract lumen centers of adjacent vessels and run into nearby irrelevant structures, e.g., the atrium and the ventricle. On average, $\approx 10$ manually clicked points are needed per data set. A way to reduce the number of anchors due to adjoining vessels has been mentioned in Section V-C2. The use of a curvilinear structure enhanced image can also eliminate the need to place anchors owing to the presence of the atrium or the ventricle.

\section{E. Clinical Data}

Figs. 14(a) and 15(a) show the major vessels extracted from the clinical data. ICA, PCoA, ACA, MCA and its branches, and PCA and its branches of the right cerebral arterial tree were extracted from the 3-D RA data set. AA, CI, internal and external CI, femoral artery (FA), and deep FA in the CT scan were delineated. The principal curves were initialized as those for BrainWeb. Figs. 14(c) and 15(c) illustrate the initial curves. The 26 vertices composed the right cerebral arterial tree; 12 of them are end vertices. The AA and its branches to the thighs were built from 12 vertices; seven of them are end vertices. A wide-neck intracranial aneurysm is at the C7 ICA segment in the 3-D RA scan. It is highlighted by the arrow in the direct volume rendered (DVR) image shown in Fig. 14(b). Our vascular model is given in Fig. 14(a). A closeup of the aneurysm region [see Fig. 14(d) for the DVR image and Fig. 14(e) for the model] illustrates that our model can bypass the pathological structure and provide a good estimation of the postembolization vessel lumen. Such a pathology-free lumen plays an important role in studying the geometry of the attached aneurysmal sac, particularly the aneurysmal neck [34]. This disease-free lumen approximation is not possible if the circular cross-sectional assumption is relaxed in our framework. Long ACA segment was extracted and modeled faithfully. Studying the planar reformatted images along its course suggested both the lumen-center locations and the widths were well estimated. Nested MCA branches were modeled without any difficulty. Due to the nonparametric representation of the principal curves, modeling of $n$-way furcation becomes possible; the MCA trifurcation shown in Fig. 14(f) is an exemplar.

Fig. 15(a) shows lumens after endovascular stent grafting extracted from the CT scan, and a DVR image is given in Fig. 15(b). Lumen centers of the two parallel artificial CI arteries were accurately localized. Jumping between abutting lumens as in other state-of-the-art methods reported in [11] will not happen in our framework because adjacent polygonal lines that model the $\mathrm{CI}$ arteries compete nearby voxels in the data point projection step. Voxels belonging to a CI artery are rarely projected onto the polygonal lines that model the other 
TABLE IV

Average Overlap and Number of Anchor Points Per Training Data Set. Please Refer to Section V-C-1 fOR the Meaning of Evaluation Metrics. The Column Rank Lists the Ranking (the Least, 1.00, and the Better) OF OuR MethoD AMONGST ALL THE 14 EVALUATED STATE-OF-THE-ART METHODS

\begin{tabular}{|c|c|c|c|c|c|c|c|c|c|c|c|c|c|c|c|}
\hline \multirow{2}{*}{$\begin{array}{c}\text { D } \\
\text { nr. }\end{array}$} & \multicolumn{3}{|c|}{ OV } & \multicolumn{3}{|c|}{$\mathbf{O F}$} & \multicolumn{3}{|c|}{ OT } & \multirow{2}{*}{$\begin{array}{l}\text { Avg. } \\
\text { rank }\end{array}$} & \multicolumn{4}{|c|}{ Anchor pts } & \multirow[b]{2}{*}{ Total } \\
\hline & $\%$ & score & rank & $\%$ & score & rank & $\%$ & score & rank & & RCA & LAD & LCX & LSB & \\
\hline 8 & 92.2 & 65.4 & 3.00 & 62.0 & 41.3 & 5.25 & 93.4 & 63.3 & 3.50 & 3.92 & 2 & 19 & 4 & 3 & 25 \\
\hline 9 & 97.9 & 70.0 & 4.00 & 80.5 & 62.6 & 6.25 & 99.0 & 82.2 & 3.25 & 4.50 & 4 & 3 & 2 & 0 & 8 \\
\hline 10 & 96.0 & 74.2 & 4.75 & 93.6 & 72.7 & 3.50 & 96.3 & 86.2 & 3.50 & 3.92 & 0 & 2 & 7 & 2 & 8 \\
\hline 11 & 95.8 & 50.5 & 4.00 & 48.6 & 37.7 & 6.00 & 95.8 & 50.5 & 4.75 & 4.92 & 0 & 6 & 1 & 3 & 6 \\
\hline 12 & 95.1 & 73.1 & 3.25 & 67.4 & 48.3 & 3.75 & 97.5 & 73.5 & 4.25 & 3.75 & 1 & 3 & 3 & 3 & 6 \\
\hline 13 & 96.0 & 73.4 & 4.00 & 76.3 & 63.9 & 4.75 & 95.9 & 73.2 & 5.00 & 4.58 & 0 & 5 & 3 & 3 & 6 \\
\hline 14 & 99.8 & 89.8 & 1.50 & 83.9 & 82.6 & 2.25 & 99.8 & 89.8 & 1.75 & 1.82 & 4 & 2 & 1 & 3 & 7 \\
\hline 15 & 100.0 & 100.0 & 1.00 & 100.0 & 100.0 & 1.00 & 100.0 & 100.0 & 1.00 & 1.00 & 2 & 3 & 3 & 3 & 6 \\
\hline 16 & 98.8 & 81.9 & 2.25 & 92.4 & 71.5 & 2.00 & 99.9 & 87.5 & 2.00 & 2.08 & 2 & 3 & 1 & 8 & 10 \\
\hline 17 & 85.6 & 68.4 & 5.00 & 75.7 & 63.0 & 3.00 & 85.6 & 68.1 & 5.25 & 4.40 & 1 & 0 & 0 & 1 & 2 \\
\hline 18 & 99.3 & 72.6 & 3.00 & 76.7 & 59.0 & 4.00 & 99.3 & 72.5 & 3.50 & 3.50 & 0 & 2 & 4 & 3 & 6 \\
\hline 19 & 98.4 & 92.6 & 2.50 & 89.0 & 83.3 & 3.75 & 98.4 & 90.5 & 2.50 & 2.92 & 0 & 2 & 1 & 2 & 2 \\
\hline 20 & 95.3 & 53.1 & 6.00 & 52.1 & 28.7 & 4.75 & 95.3 & 48.0 & 6.00 & 5.60 & 0 & 2 & 4 & 2 & 5 \\
\hline 21 & 98.4 & 81.2 & 4.00 & 97.4 & 86.2 & 3.75 & 98.4 & 80.3 & 4.25 & 4.00 & 0 & 2 & 4 & 2 & 5 \\
\hline 22 & 100.0 & 100.0 & 1.00 & 100.0 & 100.0 & 1.00 & 100.0 & 100.0 & 1.00 & 1.00 & 0 & 1 & 1 & 0 & 1 \\
\hline 23 & 99.4 & 85.1 & 3.25 & 95.3 & 73.2 & 4.25 & 99.4 & 81.8 & 3.25 & 3.58 & 3 & 3 & 4 & 5 & 11 \\
\hline 24 & 97.7 & 74.4 & 2.25 & 71.9 & 64.6 & 2.50 & 98.8 & 74.4 & 2.00 & 2.25 & 0 & 4 & 7 & 7 & 17 \\
\hline 25 & 97.5 & 61.5 & 2.25 & 65.1 & 47.7 & 3.50 & 99.2 & 74.6 & 1.50 & 2.42 & 5 & 6 & 4 & 3 & 13 \\
\hline 26 & 83.6 & 55.8 & 3.75 & 37.3 & 34.7 & 6.25 & 83.2 & 55.6 & 3.75 & 4.58 & 0 & 0 & 1 & 0 & 1 \\
\hline 27 & 88.2 & 50.6 & 5.75 & 51.5 & 29.5 & 5.75 & 88.1 & 49.6 & 6.00 & 5.85 & 7 & 9 & 1 & 2 & 16 \\
\hline 28 & 95.0 & 72.9 & 4.50 & 61.2 & 45.8 & 7.75 & 95.4 & 70.9 & 5.00 & 5.75 & 0 & 12 & 7 & 3 & 17 \\
\hline 29 & 95.3 & 72.8 & 3.75 & 73.1 & 62.2 & 3.00 & 97.1 & 73.6 & 3.75 & 3.50 & 0 & 6 & 3 & 8 & 12 \\
\hline 30 & 91.6 & 59.2 & 6.75 & 86.5 & 57.3 & 5.25 & 94.0 & 72.5 & 6.00 & 6.00 & 0 & 9 & 3 & 4 & 12 \\
\hline 31 & 92.5 & 59.9 & 8.00 & 90.7 & 58.4 & 7.25 & 96.6 & 73.4 & 4.75 & 6.65 & 0 & 9 & 1 & 2 & 9 \\
\hline Avg. & 95.4 & 72.4 & 3.73 & 76.2 & 61.4 & 4.19 & 96.1 & 74.7 & 3.65 & 3.85 & 1.3 & 4.7 & 2.9 & 3.0 & 8.8 \\
\hline
\end{tabular}

TABLE V

Average ACCuracy and Voxel Diagonal Length PER TRAINING DATA SET

\begin{tabular}{|c|c|c|c|c|c|c|c|c|c|c|c|}
\hline D & & AI & & $\mathbf{A}$ & D & D & & AI & & g. & VD \\
\hline nr. & $\mathbf{m m}$ & score & |rank & rank & $\mathbf{m m}$ & nr. & $\mathbf{m m}$ & & rank & ank & $\mathbf{m m}$ \\
\hline 8 & 0.38 & 38.0 & 8.50 & 8.50 & 1.02 & 20 & 0.38 & 35.4 & 6.75 & 6.75 & 1.04 \\
\hline 9 & 0.23 & 36.9 & 5.25 & 5.25 & 0.95 & 21 & 0.23 & 34.2 & 5.75 & 5.75 & 0.97 \\
\hline 10 & 0.29 & 33.2 & 5.75 & 5.75 & 0.87 & 22 & 0.24 & 41.1 & 3.50 & 3.50 & 1.00 \\
\hline 11 & 0.34 & 39.0 & 5.75 & 5.75 & 0.91 & 23 & 0.30 & 37.1 & 5.50 & 5.50 & 0.96 \\
\hline 12 & 0.32 & 30.9 & 7.00 & 7.00 & 1.07 & 24 & 0.23 & 35.7 & 5.00 & 5.00 & 0.84 \\
\hline 13 & 0.32 & 32.4 & 8.25 & 8.25 & 1.05 & 25 & 0.27 & 37.2 & 4.00 & 4.00 & 0.93 \\
\hline 14 & 0.30 & 39.6 & 5.75 & 5.75 & 1.03 & 26 & 0.42 & 48.2 & 5.00 & 5.00 & 0.97 \\
\hline 15 & 0.27 & 36.6 & 5.75 & 5.75 & 1.05 & 27 & 0.34 & 36.8 & 5.25 & 5.25 & 1.22 \\
\hline 16 & 0.27 & 34.7 & 6.25 & 6.25 & 0.95 & 28 & 0.29 & 29.5 & 5.50 & 5.50 & 0.97 \\
\hline 17 & 0.40 & 43.0 & 5.75 & 5.75 & 1.08 & 29 & 0.27 & 32.9 & 5.50 & 5.50 & 1.00 \\
\hline 18 & 0.28 & 34.2 & 6.50 & 6.50 & 1.00 & 30 & 0.28 & 33.0 & 5.25 & 5.25 & 0.92 \\
\hline 19 & 0.28 & 43.8 & 4.75 & 4.75 & 1.01 & 31 & 0.25 & 28.4 & 6.00 & 6.00 & 0.93 \\
\hline & & & & & & $\begin{array}{l}\text { Avg. } \\
\text { (AII) }\end{array}$ & 0.30 & 36.3 & 5.76 & \begin{tabular}{|l|}
5.76 \\
\end{tabular} & 0.99 \\
\hline
\end{tabular}

TABLE VI

SUMMARY OF THE TRAINING SET (DATA SET 0-7)

\begin{tabular}{|c|c|c|c|c|c|c|c|c|c|}
\hline & \multicolumn{3}{|c|}{$\% / \mathrm{mm}$} & \multicolumn{3}{|c|}{ score } & \multicolumn{3}{|c|}{ rank } \\
\hline & $\min$. & $\max$. & avg. & $\min$. & $\mathbf{m a}$ & |avg & $\min$. & max. & |avg. \\
\hline OV & $56.1 \%$ & $100.0 \%$ & $95.4 \%$ & 29.4 & 100.0 & 72.4 & 1 & 12 & 3.73 \\
\hline $\mathrm{OF}$ & 10 & $100.0^{\circ}$ & 76.2 & 8.1 & 100.0 & 61.4 & 1 & 13 & 4.19 \\
\hline OT & $56.1 \%$ & $100.0 \%$ & $96.1 \%$ & 28.5 & 100.0 & 74.7 & 1 & 12 & 3.65 \\
\hline $\mathrm{AI}$ & $0.16 \mathrm{~mm}$ & $0.54 \mathrm{~mm}$ & $0.30 \mathrm{~mm}$ & 21.8 & 54.0 & 36.3 & 1 & 12 & 5.76 \\
\hline Total & & & & & & & 1 & 13 & 4.81 \\
\hline
\end{tabular}

CI artery. Fig. 15(d)-(f) shows a few image slices overlaid with model outlines. Model contours adequately delineate the lumen cross sections in these slices.

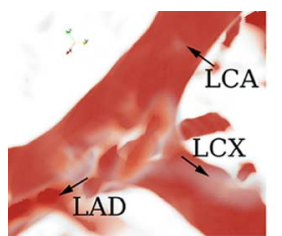

(a)

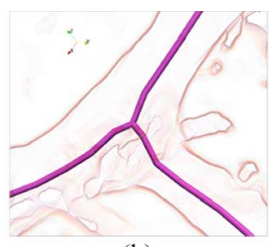

(b)

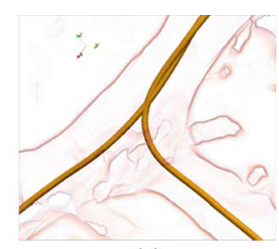

(c)
Fig. 13. Terminal bifurcation of the LCA of Data set 5 in the Rotterdam Coronary Artery database. The LCA is branched into the LAD and the LCX. (a) DVR image of the bifurcation. Lumen centers extracted with (b) our algorithm and (c) algorithm of Frangi etal [16]. There are two separate paths from the algorithm of Frangi etal, one is from LCA to LCX and the other is from LCA to LAD. On the other hand, our method produced a single arterial network. The edge image of the DVR is overlaid on top for better illustration.

\section{CONCLUSION}

We have proposed a novel approach to extract arterial lumen centers and estimate flow channel width in angiography. The method is based upon a nonlinear principal curves and an algorithm known as the polygonal line algorithm. We have described the theory of principal curves and elucidated its extension to grayscale images, proposed a practical implementation to find principal curves, and demonstrated ways to perform initialization with geodesic and minimum cost paths for reducing the amount of user interaction. A nonparametric model is used to represent lumen centers, making the modeling of a complex vascular network possible. Combining such representation and the polygonal line algorithm, we are, to the best of our knowledge, the first to develop an energy-minimization-based framework to locate the centerline and estimate the width of tubelike objects from their structural network with a nonparametric model. Our 


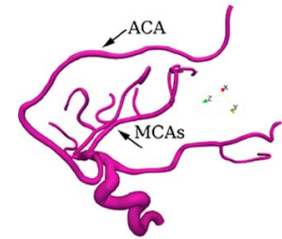

(a)

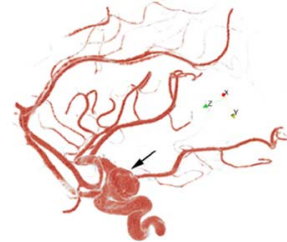

(b)

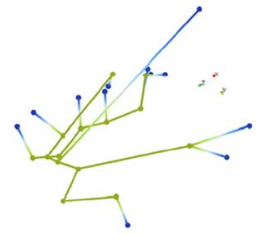

(c)

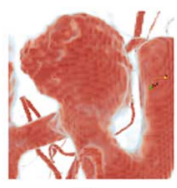

(d)

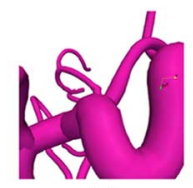

(e)

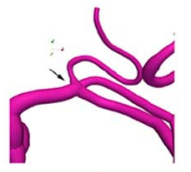

(f)
Fig. 14. Major vessels extracted from a 3-D RA of the right half of a cerebral circulation are shown. ICA, PCoA, ACA, MCA and its branches, and PCA and its branches are present. (a) Vascular model. Truncated cones [5] are used to visualize the model. (b) DVR image. (c) Initial principal curves. End vertices are in blue (dark gray); they are either inlets or outlets of the arterial network. Additional vertices are in green (light gray); they are at branching. A closeup of the aneurysmal sac is given in (d); the corresponding vascular model is present in (e) providing a good estimation of the postembolization vessel lumen. The MCA trifurcation of the vascular model is shown in (f) (a)

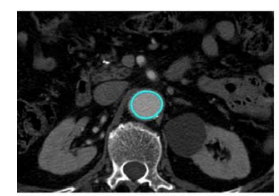

(d)

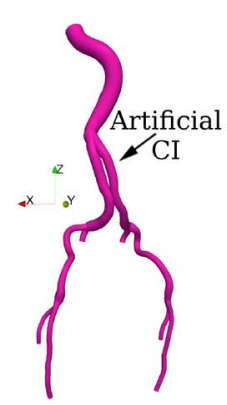

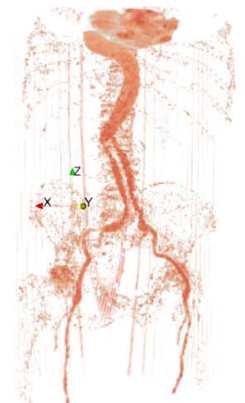

(b)

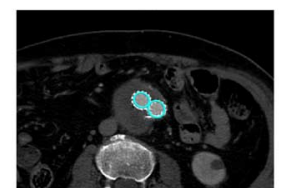

(e)

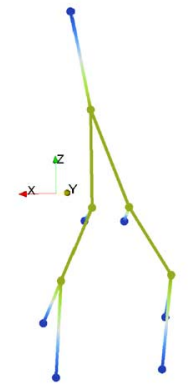

(c)

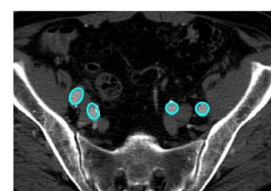

(f)
Fig. 15. Major vessels extracted from a CT scan of the abdomen and the pelvis are shown. AA, CI, internal and external CI, FA, and deep FA are present. The vascular model is given in (a); the direct volume rendered image of the intensityrescaled volume is in (b). The stent graft and bone structures are suppressed in the intensity-rescaled volume. The initial principal curves are given in (c). End vertices are in blue (dark gray) and are the inlet and outlets of the AA network. Additional vertices are in green (light gray) and are at branching. A few image slices overlaid with model outlines (in cyan/gray closed curves) are present in (d)-(f). Elliptical outlines are shown because the model penetrates the image slices at an oblique angle to the vessel axis.

vascular model is capable of depicting nested tubular branches and $n$-way furcations that are commonly found in an arterial system, particularly the cerebral vasculature. The nonparametric representation also opens an opportunity to extract arterial networks with an anatomical atlas.

We have validated the proposed method on three numerical phantoms, 19 vascular models from the BrainWeb database, and 32 clinical data sets from the Rotterdam Coronary Artery database. Rigorous validation has been conducted on the BrainWeb data sets to demonstrate the applicability of our novel method to realistic vasculatures. This is the first attempt in the literature at validating a lumen-center extraction algorithm with the BrainWeb data. Evaluation on the Rotterdam data has been performed by the organizer according to their own measures. We have also tested the method on two publicly available clinical data sets, i.e., 3-D RA and CT. Experimental results show that the lumen-center location and the flow channel width estimation accuracy is at subvoxel level. Our algorithm has high robustness to noise, INU, voxel anisotropy, and variation in image resolution. Furthermore, it provides a satisfactory estimation of the postembolization vessel lumen, which plays an important role in a geometric study of aneurysmal neck. Comparison with the closest work [16] has been performed. Potential pitfalls of that work have been revealed and discussed in this paper. Testing on two BrainWeb data sets suggests that our method outperforms its counterpart in various aspects. The proposed method can adapt to the vasculature complexity and is robust to a strongly bended lumen and branching vasculature.

\section{APPENDIX A CORRELATION-BASED PMF}

Our intensity model $h_{t}(\cdot)$ as stated in (4) is defined w.r.t. a lumen center because it takes the Euclidean distance from a point to the center as argument. Maximum correlation and, hence, probability mass are at the lumen center if the model has a finite span and the image is absent of noise. We study the behavior of the pmf $g_{\mathbf{X}}(\vec{x})$ under two noise models in medical imaging. We consider a 2-D problem in this paper for simplicity. We begin with a commonly assumed image noise zero-mean Gaussian with variance $\sigma^{2}$. Suppose $\mathbf{Y}$ is the noise random variable, $\stackrel{\circ}{\mathbf{f}}(t)$ denotes the lumen-center position, and $\hat{n}_{t}$ is the normal vector at $\stackrel{\circ}{\mathbf{f}}(t)$. We have the intensity at the position along normal direction to $\mathbf{f}(t)$, i.e.,

$$
I\left(\vec{x}+v \hat{n}_{t}\right)=h_{t}\left(\left\|\vec{x}+v \hat{n}_{t}-\stackrel{\circ}{\mathbf{f}}(t)\right\|\right)+\mathbf{Y}
$$

where $v$ is a parametric variable and $\vec{x}=\stackrel{\circ}{\mathbf{f}}(t)+u \hat{n}_{t}, \exists u$, i.e., $\stackrel{\circ}{f}^{-1}(\vec{x})=t$ and $g_{\mathbf{X}}(\vec{x})$ can be rewritten as follows. Let $H_{1}=$ $h_{t}\left(\left\|\vec{x}+v \hat{n}_{t}-\stackrel{\circ}{\mathbf{f}}(t)\right\|\right)$ and $H_{2}=h_{t}\left(\left\|v \hat{n}_{t}\right\|\right)$, then

$$
\begin{aligned}
g_{\mathbf{X}}(\vec{x}) & =\frac{1}{Z} \int_{-a}^{a}\left(H_{1}+\mathbf{Y}\right) H_{2} d v \\
& =\frac{1}{Z} \int_{-a}^{a} H_{2} H_{1} d v+\frac{1}{Z} \int_{-a}^{a} H_{2} \mathbf{Y} d v .
\end{aligned}
$$

Approximating the second integral in $g_{\mathbf{X}}$ numerically with Riemann sum, $g_{\mathbf{X}}$ follows a Gaussian distribution with mean, i.e.,

$$
\overline{g_{\mathbf{X}}}(\vec{x})=\frac{1}{Z} \int_{-a}^{a} h_{t}\left(\left\|\vec{x}+v \hat{n}_{t}-\stackrel{\circ}{\mathbf{f}}(t)\right\|\right) h_{t}\left(\left\|v \hat{n}_{t}\right\|\right) d v
$$

because the sum of weighted zero-mean Gaussian random variables is a zero-mean Gaussian random variable [35]. This mean value is the pmf value in the absence of noise, and hence, noisy 
$g_{\mathrm{X}}$ is expected to give a maximum value at the lumen center. Another property of $\overline{g_{\mathbf{X}}}(\vec{x})$ is symmetry. Consider the pmf value at $\stackrel{\circ}{\mathbf{f}}(t)-u \hat{n}, \exists u$, we find that the mean pmf is symmetric with respect to the lumen center along the normal direction, i.e.,

$$
\begin{aligned}
\overline{g_{\mathbf{X}}}(\stackrel{\circ}{\mathbf{f}}(t)-u \hat{n}) & =\frac{1}{Z} \int_{-a}^{a} h_{t}\left(\left\|v \hat{n}_{t}-u \hat{n}_{t}\right\|\right) H_{2} d v \\
& =\frac{-1}{Z} \int_{a}^{-a} h_{t}\left(\left\|-w \hat{n}_{t}-u \hat{n}_{t}\right\|\right) H_{2} d w \\
& =\frac{1}{Z} \int_{-a}^{a} h_{t}\left(\left\|w \hat{n}_{t}+u \hat{n}_{t}\right\|\right) H_{2} d w \\
& =\overline{g_{\mathbf{X}}}(\stackrel{\circ}{\mathbf{f}}(t)+u \hat{n}) .
\end{aligned}
$$

Since $h_{t}(\|\cdot\|)$ is symmetric, $h_{t}(\|\vec{x}\|)=h_{t}(\|-\vec{x}\|)$. We attempted to replicate the derivation for the Rician noise (a commonly used noise model in magnetic resonance imaging). However, there is no closed-form expression of the Rician sum distribution [36]; we have to study the pmf behavior under the Rician noise condition experimentally. We investigated various intensity models ranging from rectangle, triangle, Gaussian, parabolic, and Butterworth-shaped functions. Results show that the expected pmf's all have a shape similar to their noiseless counterparts but with the values offset. Nonetheless, their maximal and symmetric properties are well maintained.

\section{REFERENCES}

[1] A. A. Farag, M. S. Hassouna, R. Falk, and S. Hushek, Reliable fly-throughs of vascular trees Dept. Elect. Comput. Eng., Univ. Louisville, Louisville, KY, Tech. Rep., 2004.

[2] M. S. Hassouna, A. A. Farag, and R. Falk, "Differential fly-throughs (DFT): A general framework for computing flight paths," in Proc. Med. Imag. Comput. Comput. Assist. Interv., 2005, vol. 3749, Lecture Notes in Comput. Sci., pp. 654-661.

[3] E. Bullitt, K. E. Muller, I. Jung, W. Lin, and S. Aylward, "Analyzing attributes of vessel populations," Med. Imag. Anal., vol. 9, no. 1, pp. 39-49, Feb. 2005.

[4] M. Groher, F. Bender, R.-T. Hoffmann, and N. Navab, "Segmentationdriven 2D-3D registration for abdominal catheter interventions," in Proc. Med. Imag. Comput. Comput. Assist. Interv., 2007, pp. 527-535.

[5] H. K. Hahn, B. Preim, D. Selle, and H.-O. Peitgen, "Visualization and interaction techniques for the exploration of vascular structures," in Proc. IEEE Vis., 2001, pp. 395-578.

[6] S. Oeltze and B. Preim, "Visualization of vasculature with convolution surfaces: Method, validation and evaluation," IEEE Trans. Med. Imag., vol. 24, no. 4, pp. 540-548, Apr. 2005.

[7] N. Flasque, M. Desvignes, J.-M. Constans, and M. Revenu, "Acquisition, segmentation and tracking of the cerebral vascular tree on 3D MRA images," Med. Imag. Anal., vol. 5, no. 3, pp. 173-183, Sep. 2001.

[8] S. Bouix, K. Siddiqi, and A. Tannenbaum, "Flux driven automatic centerline extraction," Med. Imag. Anal., vol. 9, no. 3, pp. 209-221, Jun. 2005.

[9] P. J. Yim, P. L. Choyke, and R. M. Summers, "Gray-scale skeletonization of small vessels in magnetic resonance angiography," IEEE Trans. Med. Imag., vol. 19, no. 6, pp. 568-576, Jun. 2000.

[10] C. Florin, N. Paragios, and J. Williams, "Particle filters, a quasi-monte carlo solution for segmentation of coronaries," in Proc. Med. Imag. Comput. Comput. Assist. Interv., 2005, pp. 246-253.

[11] W. C. K. Wong and A. C. S. Chung, "Probabilistic vessel axis tracing and its application to vessel segmentation with stream surfaces and minimum cost paths," Med. Imag. Anal., vol. 11, no. 6, pp. 567-587, Dec. 2007.
[12] S. Worz and K. Rohr, "Segmentation and quantification of human vessels using a 3-D cylindrical intensity model," IEEE Trans. Image Process., vol. 16, no. 8, pp. 1994-2004, Aug. 2007.

[13] O. Wink, W. J. Niessen, and M. A. Viergever, "Multiscale vessel tracking," IEEE Trans. Med. Imag., vol. 23, no. 1, pp. 130-133, Jan. 2004.

[14] H. Li and A. Yezzi, "Vessels as 4D curves: Global minimal 4D paths to extract 3D tubular surfaces," in Proc. Comput. Vis. Pattern Recog., A. Yezzi, Ed., 2006, p. 82.

[15] W. C. K. Wong and A. C. S. Chung, "Principal curves to extract vessels in 3D angiograms," in Proc. Workshop Math. Methods Biomed. Image Anal., Jun. 2008.

[16] A. F. Frangi, W. J. Niessen, R. M. Hoogeveen, T. V. Walsum, and M. A. Viergever, "Model-based quantitation of 3-D magnetic resonance angiographic images," IEEE Trans. Med. Imag., vol. 18, no. 10, pp. 946-956, Oct. 1999.

[17] T. Hastie and W. Stuetzle, "Principal curves," J. Amer. Stat. Assoc., vol. 84, no. 406, pp. 502-516, 1989.

[18] B. Kégl, A. Krzyzak, T. Linder, and K. Zeger, "Learning and design of principal curves," IEEE Trans. Pattern Anal. Mach. Intell., vol. 22, no. 3, pp. 281-297, Mar. 2000

[19] R. Tibshirani, "Principal curves revisited," Stat. Comput., vol. 2, pp. 183-190, 1992

[20] K. Krissian, C.-F. Westin, and R. Kikinis, "Fast and automatic vessel centerline detection for MRA," in Proc. Int. Soc. Magn. Reson. Med. 10, 2002.

[21] P. Agarwal and J. Matousek, "On range searching with semialgebraic sets," Discrete Comput. Geometry, vol. 11, no. 1, pp. 393-418, Dec. 1994.

[22] M. de Berg, M. van Kreveld, M. Overmars, and O. Schwarzkopf, Computational Geometry, 2nd ed. Berlin, Germany: Springer-Verlag, 2000.

[23] McConnell Brain Imaging Centre, "BrainWeb: Simulated brain database," Montreal Neurological Institute, McGill University, 2008 [Online]. Available: http://www.bic.mni.mcgill.ca/brainweb/

[24] M. Schaap, C. Metz, T. van Walsum, and W. Niessen, "Rotterdam Coronary Artery Algorithm evaluation framework," 2009 [Online]. Available: http://coronary.bigr.nl

[25] A. P. Zijdenbos, B. M. Dawant, R. A. Margolin, and A. C. Palmer, "Morphometric analysis of white matter lesions in MR images: Method and validation," IEEE Trans. Med. Imag., vol. 13, no. 4, pp. 716-724, Dec. 1994

[26] J. J. Bartko, "Measurement and reliability: Statistical thinking considerations," Schizophr. Bull., vol. 17, no. 3, pp. 483-489, 1991.

[27] W. E. Lorensen and H. E. Cline, "Marching cubes: A high resolution 3D surface construction algorithm," Comput. Graph., vol. 21, no. 4, pp. 163-169, Jul. 1987.

[28] M. Schaap, C. Metz, T. van Walsum, and W. Niessen, "Standardized evaluation methodology and reference database for evaluating coronary artery centerline extraction algorithms," 2009 [Online]. Available: http://coronary.bigr.nl/download/evaluationframework.pdf

[29] O. Friman, C. Kühnel, and H.-O. Peitgen, "Coronary centerline extraction using multiple hypothesis tracking and minimal paths," Jul. 2008 [Online]. Available: http://coronary.bigr.nl/pdf/111_friman.pdf

[30] D. H. Douglas and T. K. Peucker, "Algorithms for the reduction of the number of points required to represent a line or its caricature," Can. Cartographer, vol. 10, no. 2, pp. 112-122, 1973.

[31] D. Kochanek and R. Bartels, "Interpolating splines with local tension, continuity and bias control," Comput. Graph., vol. 18, no. 3, pp. 33-41, Jul. 1984

[32] W. W. Esty and J. D. Banfield, The box-percentile plot Dept. Math Sci., Montana State Univ., Bozeman, MT, Tech. Rep., May 15, 1992.

[33] T. Lindeberg, "Feature detection with automatic scale selection," Int J. Comput. Vis., vol. 30, no. 2, pp. 79-116, Nov. 1998.

[34] W. C. K. Wong and A. C. S. Chung, "Augmented vessels for quantitative analysis of vascular abnormalities and endovascular treatment planning," IEEE Trans. Med. Imag., vol. 25, no. 6, pp. 665-684, Jun. 2006.

[35] A. Papoulis, Probability, Random Variables, and Stochastic Processes, 3rd ed. New York: McGraw-Hill, 1991.

[36] J. Hu and N. Beaulieu, "Accurate closed-form approximations to Ricean sum distributions and densities," IEEE Commun. Lett., vol. 9, no. 2, pp. 133-135, Feb. 2005. 


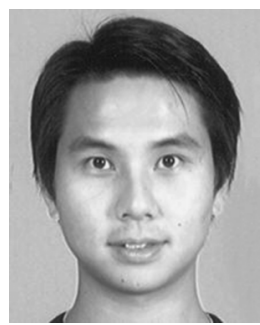

Wilbur C. K. Wong received the B.Eng. (Hons.), M.Phil., and Ph.D. degrees in computer science from Hong Kong University of Science and Technology, Clear Water Bay, Hong Kong, in 1999, 2003, and 2006, respectively.

His research interests include medical image processing and analysis.

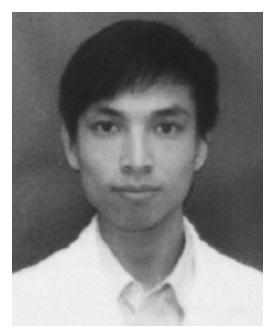

Ronald W. K. So received the B.Eng. degree (Hons.) in computer engineering and the M.Phil. degree in computer science and engineering in 2007 and 2009, respectively, from The Hong Kong University of Science and Technology, Clear Water Bay, Hong Kong, where he is currently working toward the Ph.D. degree in computer science and engineering.

His research interests include medical image processing and analysis, vascular segmentation, lumencenter extraction, and medical image registration.

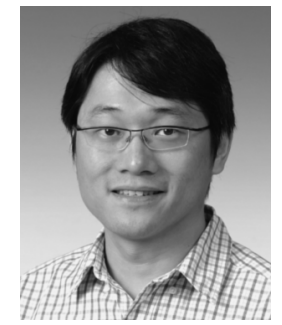

Albert C. S. Chung received the B.Eng. degree (First Class Honors) in computer engineering from The University of Hong Kong in 1995, the M.Phil. degree in computer science from The Hong Kong University of Science and Technology in 1998. He joined the Medical Vision Laboratory, University of Oxford, U.K., as a Doctoral Research Student with a Croucher Foundation scholarship and graduated in 2001.

He was a Visiting Scientist with the Artificial Intelligence Laboratory, Massachusetts Institute of Technology, Cambridge, in 2001. He is currently an Associate Professor with the Department of Computer Science and Engineering, The Hong Kong University of Science and Technology, Clear Water Bay, Hong Kong. His research interests include medical image processing and analysis, and computer vision.

Dr. Chung was the recipient of the 2002 British Machine Vision Association Sullivan Thesis Award for the best doctoral thesis submitted to a U.K. university in the field of computer or natural vision. 